\title{
Soft-bound Interval Control System and its Robust Fault-tolerant Controller Design
}

\author{
Jinglin Zhou Member, IEEE and Hong Yue, Senior Member, IEEE
}

\begin{abstract}
In this work, a soft-bound interval control problem is proposed for general non-Gaussian systems with the aim to control the output variable within a bounded region at a specified probability level. To find a feasible solution to this challenging task, the initial soft-bound interval control problem has been transformed into an output probability density function (PDF) tracking control problem with constrained tracking errors, thereby the controller design can be handled under the established framework of stochastic distribution control (SDC). Fault tolerant control has been developed for soft-bound interval control systems in presence of faults. Three fault detection methods have been proposed based on criteria extracted from the initial soft-bound control problem and the recast PDF tracking problem. An integrated design for fault estimation and fault tolerant control (FTC) is proposed based on a double proportional integral (PI) structure. This integrated FTC is developed through linear matrix inequality (LMI). Extensive simulation studies have been conducted to examine key design factors, implementation issues and effectiveness of the proposed approach.
\end{abstract}

Index Terms-Non-Gaussian systems, soft-bound control, stochastic distribution control (SDC), probability density function (PDF), fault detection, fault tolerant control (FTC).

\section{INTRODUCTION}

Stochastic control has been an active area in control engineering and applications since 1970's as most practical systems have stochastic characteristics. Continuous efforts have been made in development of minimum variance control [1] [3], linear quadratic Gaussian (LQG) control [4], Markovian stochastic control [5], stochastic adaptive control, stochastic optimization and forecasting, sliding mode control [6]-[8], to name a few. Most of these methods are focused on stochastic features of system variables, mean and variance for example, under the assumption of Gaussian distribution. In real applications, however, a large number of stochastic processes are non-Gaussian, examples include molecular weight distribution control in polymerization [9], [10], pulp fiber length distribution control in paper industries [11], particulate process control in powder industries [12], crystal size distribution control in crystallization [13], soil particle distribution control [14], flame temperature distribution control in furnace systems [15], [16] and power probability density function control in nuclear reactors [17], among others. For these systems new

This work was supported in part by Chinese NSFC (Grant No. 61473025) and UK EPSRC Project (EP/N006224/1).

J. Zhou is with the College of Information Science and Technology, Beijing University of Chemical Technology, Beijing, 100029, China. (email:jinglinzhou@mail.buct.edu.cn).

H. Yue is with the Department of Electronic and Electrical Engineering, University of Strathclyde, Glasgow G1 1XW, United Kingdom. (email:hong.yue@strath.ac.uk). Corresponding author

approaches need to be developed to control the full shape of the system output(s), which is equivalent to directly control the output probability density function (PDF) under general non-Gaussian assumption. The latter is also called output PDF shaping control or output stochastic distribution control (SDC) in literature [11], [18], [19].

Various output PDF control algorithms have been developed such as optimal tracking control [20], minimum entropy control [21], robust PDF tracking control [22], [23] and predictive PDF control [24]. Most of these controllers are designed to drive the output PDF towards a target PDF as close as possible, which can be taken as an output PDF tracking problem. Without considering the control cost, a typical performance index for PDF tracking problem can be formulated with the following index

$$
J(k)=\int_{a}^{b}\left(\gamma(y, u(k))-\gamma_{g}(y)\right)^{2} d y,
$$

where $\gamma(y, u(k))$ is the output PDF with its random variable, $y$, defined on $[a, b] ; \gamma_{g}(y)$ is the desired or target PDF defined on the same region of $[a, b]$ and it is independent of $u(k)$; $u(k)$ is the vector of control inputs; $k$ is the time index.

While controlling the output PDF may fully determine the output distribution, it is also crucial to control the output variable itself. Following operational requirements, the process outputs, $v(k)$, can be classified into two broad categories in control [25]: (i) outputs to be controlled at desired values or set-points, and (ii) outputs to be controlled within desired intervals (also called zone control). For stochastic systems, the output variables are stochastic terms, a natural choice is to control the output within a specified region with a desired probability. This interval control can be described as

$$
J_{0}(k)=P\left\{a_{0} \leq v(k) \leq b_{0}, u(k)\right\} \geq P_{0},
$$

where $a \leq a_{0}<b_{0} \leq b$, and $P_{0}$ is a pre-specified probability level. This control problem is similar to control the output variable with a soft-bound constraint [25]-[28]. For a Gaussian system, it can also be taken as a generalization of the output within the region of $[\mu-3 \sigma, \mu+3 \sigma]$ with over $99 \%$ probability for example ( $\mu$ and $\sigma$ are mean and standard deviation). Here we call the problem with performance function in (2) softbound interval control. The word 'soft bound' is used in comparison to the 'hard bound' interval control that controls the output to stay within a region under all circumstances. In (2), the $\left[a_{0}, b_{0}\right]$ interval is the soft-bound region and $P_{0}$ is the required or expected soft-bound probability level to be achieved through control actions. In practice, both the soft- 
bound region and the level of soft-bound probability should be determined following system or process requirements.

This work is focused on the soft-bound interval control of non-Gaussian systems. It is not a trivial task to find the optimal solution to this problem. One major contribution of this work is to propose an effective method that transforms the softbound interval control into an output PDF tracking problem with constrained tracking errors. The latter can be solved through our previous results under the output SDC framework. We therefore call this new strategy soft-bound PDF tracking control.

Another key exploration through this work is to investigate fault detection and diagnosis (FDD) and fault-tolerant control (FTC) for soft-bound interval control systems that may contain faulty signals. This will help to improve reliability, security and economical efficiency of the controlled systems. Numerous methodologies for FDD and FTC have been established [29]-[33]. A well-developed technique for model-based FDD and FTC relies on its analytical redundancy in the form of dedicated observers [34]-[40]. Most current FDD and FTC algorithms for stochastic systems are developed for Gaussian processes, with only very few results for general non-Gaussian SDC systems. In a SDC system, the purpose of FDD is to use information on the control input and the output PDF to determine whether a fault occurs, and to estimate and locate the fault. Observer-based (filter-based) methods are often used in FDD, where it is crucial to generate residual signals that are robust to unknown inputs but sensitive to fault signals [41][46]. In [47], an observer is designed via the use of linear matrix inequalities (LMIs) and the fault detection threshold is determined by the bounds imposed on model uncertainties. A nonlinear adaptive observer-based fault diagnosis algorithm [48] and an iterative learning observer-based fault diagnosis algorithm [44] are employed for normal and singular nonGaussian systems, respectively.

Back to the novel idea of soft-bound interval control, fault detection for Gaussian systems is relatively straightforward that can be realized from the analysis of output data without designing a filter. An over $99 \%$ level is commonly used as the detection threshold, which corresponds to the probability that the Gaussian distributed variable falls within the region of $[\mu-3 \sigma, \mu+3 \sigma]$. For non-Gaussian SDC systems, however, one question is whether a fault can be detected by a probability threshold (or any other given threshold)? If yes, how such a threshold can be determined from the output stochastic distribution information? Is it necessary to develop a separate fault diagnosis observer (filter) for FTC in soft-bound control systems? These questions will be discussed in this work. A new design of integrated FDD and FTC for soft-bound PDF tracking makes another major contribution of this work.

The remaining of the paper is organized as follows. In Section II, the soft-bound interval control problem is recast into output PDF tracking control with constrained errors. A structured proportional integral (PI) robust controller is developed through LMI for fault-free systems in Section III. For soft-bound output control systems in presence of faults, three fault detection methods are proposed, based on which an integrated design of FDD and FTC is proposed with a double-PI structured robust controller in Section IV. Simulation studies are conducted in Section V to examine the feasibility, effectiveness and key design factors of the proposed algorithm. Conclusions and discussions are given in Section VI. Theoretical proof of lemmas and theorems are provided in appendix.

\section{Soft-Bound Output Control And Constrained PDF TRACKING}

\section{A. Modeling of Output PDFs}

For a dynamic stochastic control system, denote $v(k) \in$ $[a, b]$ as the random output and $u(k) \in \mathbb{R}^{q \times 1}$ as the control input vector. At time $k$, the distribution of $v(k)$ can be characterized by its PDF, $\gamma(y, u(k))$. The probability that $v(k)$ locates in the range of $[a, \zeta]$ under control $u(k)$ is represented by

$$
P\{a \leq v(k) \leq \zeta, u(k)\}=\int_{a}^{\zeta} \gamma(y, u(k)) d y .
$$

Using the square root B-spline approximation [11], the PDF of the output variable can be represented by

$$
\sqrt{\gamma(y, u(k))}=\sum_{i=1}^{n} w_{i}(u(k)) B_{i}(y)+e_{0}(y, u(k)),
$$

in which $B_{i}(y)(i=1,2, \cdots n)$ are the $n$ pre-specified basis functions defined on the interval $[a, b], w_{i}(u(k))$ are the corresponding weights dependent on $u(k)$. This square-root Bspline model guarantees positiveness in PDF approximation. Since the integration constraint of $\int_{a}^{b} \gamma(y, u(k)) d y=1$ is required for all PDFs, only $(n-1)$ weights are independent in this B-spline model. The PDF approximation errors, $e_{0}(y, u)$, can be considered as modeling uncertainty as shown later on. To start with, dropping the error term for simplicity, (4) can be rewritten into a compact form as

$$
\sqrt{\gamma(y, u(k))}=C(y) V(k)+H(V(k)) B_{n}(y),
$$

where $C(y)=\left[B_{1}(y), B_{2}(y), \cdots, B_{n-1}(y)\right]$ is the vector of independent basis functions, and $V(k)=\left[w_{1}(u(k))\right.$, $\left.w_{2}(u(k)), \cdots, w_{n-1}(u(k))\right]^{\mathrm{T}}$ is the vector of the corresponding weights. Denote

$$
\begin{aligned}
\Phi_{1} & =\int_{a}^{b} C^{\mathrm{T}}(y) C(y) d y \\
\Phi_{2} & =\int_{a}^{b} C(y) B_{n}(y) d y \\
\Phi_{3} & =\int_{a}^{b} B_{n}^{2}(y) d y .
\end{aligned}
$$

Following the PDF integration constraint of $\int_{a}^{b} \gamma(y, u(k)) d y=$ 1 , it can be derived from (5) that

$$
H(V(k))=\frac{ \pm \sqrt{\Phi_{3}-V^{\mathrm{T}}(k) \Phi_{0} V(k)}-\Phi_{2} V(k)}{\Phi_{3}},
$$

where $\Phi_{0}=\Phi_{1} \Phi_{3}-\Phi_{2}^{\mathrm{T}} \Phi_{2}$. For simplify, only the "+" in (7) is considered in the rest of the paper. Denoting $\Sigma=\Phi_{1}$ $\Phi_{3}^{-1} \Phi_{2}^{\mathrm{T}} \Phi_{2}$, from (7), the following inequality

$$
V^{\mathrm{T}}(k) \Sigma V(k) \leq 1
$$


needs to be satisfied. This constraint on $V(k)$ makes the output PDF tracking controller design more complicated [47]. Under inequality (8), we have Lemmas 1 and 2 stated in the following.

Lemma 1: For a function

$f\left(V\left(k_{1}\right), V\left(k_{2}\right)\right)=\sqrt{V^{\mathrm{T}}\left(k_{1}\right) \Phi_{0} V\left(k_{1}\right)}-\sqrt{V^{\mathrm{T}}\left(k_{2}\right) \Phi_{0} V\left(k_{2}\right)}$, it has a $\lambda=\frac{\lambda_{\max }\left(\Phi_{0}\right)}{\sqrt{\lambda_{\min }\left(\Phi_{0}\right)}}$ such that

$$
\left\|f\left(V\left(k_{1}\right), V\left(k_{2}\right)\right)\right\| \leq \lambda\|\| V\left(k_{1}\right)\|-\| V\left(k_{2}\right)\|\| .
$$

Lemma 1 and its proof can be found in reference [24]. This Lemma is introduced to prove Lemma 2 presented as follows.

Lemma 2: For the given $V\left(k_{1}\right)$ and $V\left(k_{2}\right)$ in (8), there exist $M_{\max }$ and $M_{\min }$ such that

$$
\begin{aligned}
\left\|H\left(V\left(k_{1}\right)\right)-H\left(V\left(k_{2}\right)\right)\right\| & \leq M_{\max }\left\|V\left(k_{1}\right)-V\left(k_{2}\right)\right\| \\
\left\|H\left(V\left(k_{1}\right)\right)+H\left(V\left(k_{2}\right)\right)\right\| & \geq M_{\min }\left\|V\left(k_{1}\right)+V\left(k_{2}\right)\right\|
\end{aligned}
$$

hold. In particular, when

$$
V^{\mathrm{T}}\left(k_{1}\right) \Phi_{0} V\left(k_{1}\right)+V^{\mathrm{T}}\left(k_{2}\right) \Phi_{0} V\left(k_{2}\right) \leq \Phi_{3},
$$

there are

$$
\begin{aligned}
& M_{\max }=\frac{\frac{\lambda_{\max }\left(\Phi_{0}\right)}{\sqrt{\lambda_{\min }\left(\Phi_{0}\right)}}+\left\|\Phi_{2}\right\|}{\left\|\Phi_{3}\right\|} \\
& M_{\min }=\frac{\left\|\sqrt{\lambda_{\min }\left(\Phi_{0}\right)}-\right\| \Phi_{2}\|\|}{\left\|\Phi_{3}\right\|}
\end{aligned}
$$

where $\lambda_{\max }\left(\Phi_{0}\right)$ and $\lambda_{\min }\left(\Phi_{0}\right)$ are the maximum and the minimum eigenvalues of $\Phi_{0}$, respectively.

Proof: See Appendix A.

\section{B. Output PDF Tracking Control with Constrained Errors}

With the use of PDF, the soft-bound output control objective in (2) can be written as

$$
\int_{a_{0}}^{b_{0}} \gamma(y, u(k)) d y \geq P_{0} .
$$

For a Gaussian system, the output PDF can be determined by its mean value $(\mu)$ and the standard deviation $(\sigma)$, therefore, the soft-bound output control can be realized by controlling these two parameters to the settings of $\left(\mu_{g}, \sigma_{g}\right)$ that correspond to $P_{0}$ and accordingly a target PDF, $\gamma_{g}(y)$. This means under Gaussian assumptions, the soft-bound output control problem can be transformed into an output PDF tracking problem with the perfect tracking performance (zero tracking errors). For a general non-Gaussian system, however, its output PDF may not be explicitly determined by several parameters. It is not always possible to find an exact target PDF that would lead to a solution to (10) through an equivalent perfect (output) PDF tracking control. Next we will discuss how to choose a suitable target PDF so that the soft-bound output control objective can be achieved via output PDF tracking control with constrained tracking errors.

To keep the modeling consistency, the target PDF is also approximated by the same square-root B-spline model in (5), therefore

$$
\sqrt{\gamma_{g}(y)}=C(y) V_{g}+H\left(V_{g}\right) B_{n}(y)
$$

where $V_{g}$ is the corresponding weights vector for the target PDF, $\gamma_{g}(y)$. The integration of $\gamma_{g}(y)$ over the soft bound region gives a probability, $P_{1}$, i.e.

$$
P_{1}=\int_{a_{0}}^{b_{0}} \gamma_{g}(y) d y=\int_{a_{0}}^{b_{0}}\left(C(y) V_{g}+H\left(V_{g}\right) B_{n}(y)\right)^{2} d y .
$$

In general, $P_{1}$ needs to be greater than $P_{0}$. The difference or closeness between the two probability levels is defined as

$$
\alpha_{0}=P_{1}-P_{0} .
$$

We call $\alpha_{0}$ 'the probability discrepancy factor' for soft-bound output control. This is a key factor that affects the controller design.

An output PDF tracking control performance index is formulated following the square root B-spline approximation,

$$
\begin{aligned}
J_{1}(k) & =\int_{a}^{b}\left(\sqrt{\gamma(y, u(k))}-\sqrt{\gamma_{g}(y)}\right)^{2} d y \\
& =2-2 \int_{a}^{b} \sqrt{\gamma(y, u(k)) \gamma_{g}(y)} d y .
\end{aligned}
$$

Remark 1: The PDF tracking performance index in (14) is dependent on the the coupling of the output PDF and the target PDF. Apparently, when $P_{1}=P_{0}$, the soft-bound output control problem is equivalent to seeking $J_{1}=0$ or $\gamma(y, u(k))=$ $\gamma_{g}(y)$, which is a perfect PDF tracking for the SDC system [11]. When $P_{1} \neq P_{0}$, the soft-bound output control problem cannot be equivalent to a perfect PDF tracking control, instead, the PDF tracking errors will present.

Remark 2: It can be revealed from (14) and Lemma 2 that a good choice of the weight vector $V_{g}$ (corresponding to the target PDF $\left.\gamma_{g}(y)\right)$ is to make $V_{g}^{\mathrm{T}} \Phi_{0} V_{g}$ stay far away from $\Phi_{3}$ under the Lemma 2 requirement. If $V_{g}^{\mathrm{T}} \Phi_{0} V_{g}$ is chosen to be very close to $\Phi_{3}$, it will leave rather limited room for controller design. With a proper chosen $V_{g}$, the controller design should also ensure other constraints relevant to $M_{\max }$ and $M_{\min }$, such as $V_{g}^{\mathrm{T}} \Phi_{0} V_{g}+V^{\mathrm{T}}(k) \Phi_{0} V(k) \leq \Phi_{3}$. In this case, a variable structure strategy [20] could be a proper choice for controller design.

When a target PDF is given, under the soft-bound output control objective (10), the output PDF tracking error, measured by (14), will also be a bounded term as discussed through the following theorem.

Theorem 1: Consider a SDC system with its output PDF described by (5) and the soft-bound output control requirement in (10). Given a target PDF, modeled by (11), the instant output PDF tracking performance in (14) is bounded as follows

$$
J_{1}(k)=\int_{a}^{b}\left(\sqrt{\gamma(y, u(k))}-\sqrt{\gamma_{g}(y)}\right)^{2} d y \leq \alpha_{1}
$$

where

$$
\alpha_{1}=\min \left\{\|\Phi\| \theta_{1}^{2}\left(\alpha_{0}\right),\|\Phi\| \theta_{2}^{2}\left(\alpha_{0}\right)\right\}
$$

and

$$
\begin{aligned}
& \theta_{1}=\frac{\left\|V_{g}\right\|\left\|\Phi_{\min }\right\|-\sqrt{\left\|V_{g}\right\|^{2}\left\|\Phi_{\min }\right\|^{2}-\alpha_{0}\left\|\Phi_{\min }\right\|}}{\left\|\Phi_{\min }\right\|} \\
& \theta_{2}=\frac{\sqrt{\left\|V_{g}\right\|^{2}\left\|\Phi_{\min }\right\|^{2}+\alpha_{0}\left\|\Phi_{\min }\right\|}-\left\|V_{g}\right\|\left\|\Phi_{\min }\right\|}{\left\|\Phi_{\min }\right\|}
\end{aligned}
$$


with $\left\|\Phi_{\min }\right\|=\left\|\Phi_{01}\right\|+2\left\|M_{\min }\right\|\left\|\Phi_{02}\right\|+\left\|M_{\min }^{2}\right\|\left\|\Phi_{03}\right\|$, $\Phi_{01}=\int_{a_{0}}^{b_{0}} C^{\mathrm{T}}(y) C(y) d y, \Phi_{02}=\int_{a_{0}}^{b_{0}} C(y) B_{n}(y) d y, \Phi_{03}=$ $\int_{a_{0}}^{b_{0}} B_{n}^{2}(y) d y,\|\Phi\|=\left\|\Phi_{1}\right\|+2\left\|\Phi_{2}\right\|\left\|M_{\max }\right\|+\left\|M_{\max }\right\|^{2}\left\|\Phi_{3}\right\|$. Proof: See Appendix B.

Remark 3: As shown in Theorem 1, when the soft-bound output control is transformed into a PDF tracking control following a (chosen) target PDF, the PDF tracking error is guaranteed to be bounded. The bound is determined by the probability discrepancy level, $\alpha_{0}$, and the shape of the target PDF, $V_{g}$. For the same $V_{g}$, the larger is $\alpha_{0}$, the larger is the constraint bound of the PDF tracking errors.

We can take this bounded PDF control problem as a special case of the conventional output PDF control, in which the control objective is to make the output PDF stay "as close as possible" to the target PDF. The bounded PDF tracking control problem is formulated as follows.

$$
\begin{array}{ll}
\min & J(u(k))=\int_{a}^{b}\left(\sqrt{\gamma(y, u(k))}-\sqrt{\gamma_{g}(y)}\right)^{2} d y \\
\text { s.t. } & J(u(k)) \leq \alpha_{1}, k \rightarrow \infty, \text { and } \\
& V^{\mathrm{T}}(k) \Sigma V(k)=\|V(k)\|_{\Sigma} \leq 1
\end{array}
$$

Different from the conventional "as close as possible" PDF tracking control, this new control problem contains two constraints: one is the square-root B-spline PDF modeling constraint raised in (8), the other is the steady-state constraint for the PDF tracking performance.

\section{Structured Robust Tracking Controller DESIGN}

\section{A. Formulation of the Constrained PDF Tracking Control Problem}

Using the B-spline PDF modeling, the PDF tracking error can also be measured by the errors between weights vectors corresponding to the output PDF and the target PDF, i.e.,

$$
e(k)=V(k)-V_{g}=\left[e_{1}(k), e_{2}(k), \cdots e_{n-1}(k)\right]^{\mathrm{T}} .
$$

For simplicity but without losing any key characteristics of the soft-bound output control under discussion, the following linear model is assumed for the weights dynamics, in which an additive term, $\omega(k)$, is introduced to accommodate disturbance, model uncertainties and/or output PDF approximation errors.

$$
V(k+1)=A_{0} V(k)+B_{0} u(k)+E_{0} \omega(k)
$$

$A_{0}, B_{0}$ and $E_{0}$ are known coefficient matrices with compatible dimensions that can be established from data-based modeling. With (19), the weights tracking error in (18) can be further written as

$$
e(k+1)=A_{0} e(k)+B_{0} u(k)+\left(A_{0}-I\right) V_{g}+E_{0} \omega(k) .
$$

The purpose of controller design is to determine the control inputs, $u(k)$, such that the output PDF follows a pre-specified target PDF, $\gamma_{g}(y)$, with an $\alpha_{0}$-related upper bound on $e(k)$. Denoting $U(k)$ as

$$
B_{0} U(k)=\left(A_{0}-I\right) V_{g}+B_{0} u(k),
$$

this control problem is equivalent to making $\sqrt{\gamma(y, U(k))} \quad 296$ follow $\sqrt{\gamma_{g}(y)}$ with an upper bound on the tracking error.

Taking the two PDFs in (5) and (11) into the performance index in (17), there is

$$
\begin{gathered}
J(U(k))=\int_{a}^{b}\left(\sqrt{\gamma(y, U(k))}-\sqrt{\gamma_{g}(y)}\right)^{2} d y \\
=\int_{a}^{b}\left[\left(H(V(k))-H\left(V_{g}\right)\right) B_{n}(y)\right. \\
\left.\quad+C(y)\left(V(k)-V_{g}\right)\right]^{2} d y
\end{gathered}
$$

The performance index in (22) consists of two parts: one is a linear function of $V(k)$; the other is regarding the nonlinear term $H(V(k))$ which is a continuous function with respect to $V(k)$ as defined in (7). Following Lemma 2 and the continuity nature of function $H(V(k)),\left\|H(V(k))-H\left(V_{g}\right)\right\|$ and $\left\|V(k)-V_{g}\right\|$ have the same minimum point in optimization when $V(k)=V_{g}$. This suggests that the problem of minimizing $J(U(k))$ in (22) can be realized through minimizing $\left(C(y)\left(V(k)-V_{g}\right)\right)^{2}$ alone.

The performance index in (22) is in fact bounded by

$$
\int_{a}^{b}\left(\sqrt{\gamma(y, U(k))}-\sqrt{\gamma_{g}(y)}\right)^{2} d y \leq\|e(k)\|^{2}\|\Phi\| .
$$

This gives one constraint as

$$
\|e(k)\|^{2}\|\Phi\| \leq \alpha_{1} .
$$

The PDF integration constraint for $e(k)$ can be developed from (8) to give

$$
\left\|e(k)+V_{g}\right\|_{\Sigma} \leq 1 .
$$

The two constraints in (23) and (24) can be combined into a single constraint in the form of

$$
\|e(k)\|^{2}\|\Phi\|<\alpha_{2}, \quad k \rightarrow \infty
$$

where

$$
\alpha_{2}=\min \left\{\alpha_{1},\left(1-\left\|V_{g}\right\|_{\Sigma}\|\Phi\| /\|\Sigma\|\right)\right\} .
$$

Therefore, the constrained PDF tracking control problem can be transformed into the following optimization problem,

$$
\begin{array}{lrl}
\min & J(U(k))= & e^{\mathrm{T}}(k+1) \bar{\Lambda} e(k+1) \\
\text { s.t. } & e(k+1)= & A_{0} e(k)+B_{0} u(k)+\left(A_{0}-I\right) V_{g} \\
& +E_{0} \omega(k) ; \\
& \\
& \|e(k)\|^{2}\|\Phi\| & <\alpha_{2}
\end{array}
$$

where $\bar{\Lambda}>0$ is a given (weighting) matrix and in most cases can be chosen as $\bar{\Lambda}=\Phi_{0}$.

Remark 4: The original soft-bound output control problem is stated in (10) with the probability level of $P_{0}$ set up for the control objective. This control problem is then transformed to the bounded PDF tracking problem as described in (17) with two constraints on the performance index and the PDF integration, respectively. The integration of the target PDF over the soft-bound region is $P_{1}$ that can be calculated by (12). The difference between $P_{0}$ and $P_{1}$ is defined as the probability discrepancy factor, $\alpha_{0}$, which is used to determine the constraint for PDF tracking errors. Taking the PDF tracking error $e(k)$ as the states and considering the uncertainty term 
$\omega(k)$, the dynamic model is further represented by (20), in which the control action is denoted by $U(k)$ as in (21). Accordingly, the two constraints are re-written and combined into a single constraint as in (constraint3), which is used in controller design as the constraint level for PDF tracking errors in terms of $e(k)$. The final constrained optimization problem is given in (27).

Algorithm 1 The following procedure is provided for implementation of this soft-bound control algorithm step by step.

i) Set up the soft-bound region, $\left[a_{0}, b_{0}\right]$, and the desired probability level, $P_{0}$, as described in the soft-bound output control objective in (10).

ii) Establish the dynamic model for output PDF, $\gamma(y, u(k))$, using the square-root B-spline approximation. The compact form of the model is shown in (5). Calculate $\|\Phi\|$ as discussed in Theorem 1.

iii) Establish the constraint on PDF integration as shown in (8).

iv) Choose a target output PDF, $\gamma_{g}(y)$, and establish the Bspline approximation model in (11) for the target output PDF. Calculate the probability level $P_{1}$ by (12).

v) Calculate the probability discrepancy factor $\alpha_{0}$ by (13).

vi) Determine the bound for the output PDF tracking error, $\alpha_{1}$, following (16) in Theorem 1.]

vii) Considering the tracking error term $e(k)$ in (18), establish $A_{0}, B_{0}$ and $E_{0}$ through parameter estimation using collected input and output data, or simply take given information if known. This will set up the error dynamic model in (20).

viii) Calculate $\alpha_{2}$ with (26) for the combined constraint in (25).

ix) Set up the weighting matrix $\bar{\Lambda}$ in the performance index, solve the constrained optimization problem in (27) to obtain the optimal control action, $U(k)$. Note here $U(k)$ is introduced in (21) for the error dynamic model.

It can be seen from the above procedures that with steps i) to vi), the soft-bound output control problem in (10) has been recast into a constrained output PDF tracking problem (17). With further steps in vii) and ix), the optimization problem in (17) has been transferred to the constrained optimisation in (27) considering the PDF tracking error as variables to be controlled.

\section{B. Structured PI Controller Design via LMI}

For most SDC problems with an instant PDF tracking performance index, only numerical solutions can be developed for control input [47]. This can be inconvenient for analysis of control performance such as closed-loop stability and robustness. It would be advantageous to design a structured controller for the proposed soft-bound PDF tracking problem.

For the constrained PDF tracking control problem in (27), the following generalized PI control structure is proposed

$$
\begin{aligned}
U(k) & =K_{P_{0}} \varepsilon(k)+K_{I_{0}} \nu(k) \\
\nu(k+1) & =\nu(k)+T_{0} \varepsilon(k) \\
\varepsilon(k) & =\int_{a}^{b}\left(\sqrt{\gamma(y, U(k))}-\sqrt{\gamma_{g}(y)}\right) d y
\end{aligned}
$$

where $K_{P_{0}}$ and $K_{I_{0}}$ are the proportional and integral gain matrices, $\varepsilon(k)$ is an integral term that reflects the output PDF tracking error at time $k$. The controller design task is to find $K_{P_{0}}$ and $K_{I_{0}}$ to solve the constrained optimization problem.

Denote $x_{S}(k)=\left[e^{\mathrm{T}}(k), \nu^{\mathrm{T}}(k)\right]^{\mathrm{T}}$ and

$$
h(k)=H(V(k))-H\left(V_{g}\right),
$$

the following augmentation system can be constructed

$$
x_{S}(k+1)=A_{S} x_{S}(k)+B_{S} h(k)+E_{S} w(k),
$$

where

$$
\begin{aligned}
& A_{S}=\left[\begin{array}{cc}
A_{0}+B_{0} K_{P_{0}} \Sigma_{0} & B_{0} K_{I_{0}} \\
T_{0} \Sigma_{0} & I
\end{array}\right], \\
& B_{S}=\left[\begin{array}{c}
B_{0} K_{P_{0}} \Sigma_{1} \\
T_{0} \Sigma_{1}
\end{array}\right], \quad E_{S}=\left[\begin{array}{c}
E_{0} \\
0
\end{array}\right] .
\end{aligned}
$$

Here $\Sigma_{0}=\int_{a}^{b} C^{\mathrm{T}}(y) d y, \Sigma_{1}=\int_{a}^{b} B_{n}^{\mathrm{T}}(y) d y$. The following theorem provides a solution to the constrained PDF tracking control problem with the proposed PI control structure.

Theorem 2: With the known parameters, $\lambda, \mu_{1}, \mu_{2}$ and matrix $M_{\max }$, suppose that there exist $\Lambda>0$ and $K_{0}=$ $\left[K_{P_{0}}, K_{I_{0}}\right]$ such that the following LMI is solvable,

$$
\left[\begin{array}{cccc}
\Psi_{0} & 0 & 0 & A_{S_{0}}^{\mathrm{T}} \Lambda+A_{S_{1}}^{\mathrm{T}} R \\
* & -\lambda^{2} I & 0 & B_{S_{0}}^{\mathrm{T}} \Lambda+B_{S_{1}}^{\mathrm{T}} R \\
* & * & -\mu_{1}^{2} I & E_{S}^{\mathrm{T}} \Lambda \\
* & * & * & -\Lambda
\end{array}\right]<0
$$

in which

$$
\begin{aligned}
\Psi_{0} & =-\Lambda+\mu_{2}^{2} T+\lambda^{2} M_{\max }^{\mathrm{T}} M_{\max } \\
T & =\operatorname{diag}\{\Phi, 0\}
\end{aligned}
$$

and

$$
\begin{array}{rlrl}
A_{S_{0}} & =\left[\begin{array}{cc}
A_{0} & 0 \\
T_{0} \Sigma_{0} & I
\end{array}\right], & A_{S_{1}} & =\left[\begin{array}{cc}
\Sigma_{0}^{\mathrm{T}} \Sigma_{1}^{-1} & 0 \\
0 & I
\end{array}\right] \\
B_{S_{0}} & =\left[\begin{array}{c}
0 \\
T_{0} \Sigma_{1}
\end{array}\right], & B_{S_{1}} & =\left[\begin{array}{l}
I \\
0
\end{array}\right] \\
\Lambda & =\left[\begin{array}{ll}
\Lambda_{1} & \Lambda_{2}
\end{array}\right]^{\mathrm{T}} & R & =\left[\begin{array}{ll}
r_{1} & r_{2}
\end{array}\right]^{\mathrm{T}}
\end{array}
$$

then the closed-loop system (30) is stable and satisfies $e^{\mathrm{T}}(k) \Phi e(k)<\mu_{2}^{-2} \mu_{1}^{2}\|\omega(k)\|^{2}$.

Proof: The proof of this Theorem is similar to the proof of Theorem 3, the latter is detailed in Appendix C.

In this case, the PI control gains, $K_{P_{0}}$ and $K_{I_{0}}$, can be solved via $r_{1}=\Sigma_{1}^{\mathrm{T}} K_{P_{0}}^{\mathrm{T}} B_{0}^{\mathrm{T}} \Lambda_{1}$ and $r_{2}=K_{I_{0}}^{\mathrm{T}} B_{0}^{\mathrm{T}} \Lambda_{2}$, respectively. When appropriate values for $\mu_{1}$ and $\mu_{2}$ are selected such that $\alpha_{2} \geq \mu_{2}^{-2} \mu_{1}^{2}\|\omega(k)\|^{2}$, the PDF tracking control performance can be achieved at $k \rightarrow \infty$. The PI-structured robust controller (28) will be expanded to FTC design for soft-bound PDF tracking next in Section IV.

\section{Fault Detection and Fault-Tolerant Tracking} CONTROL DESIGN

\section{A. Fault Detection Methods Based On Output PDF Data}

Assume that the faulty system can be expanded from model (19) as,

$$
V(k+1)=A_{0} V(k)+B_{0} u(k)+E_{0} \omega(k)+G F(k),
$$


where $F(k)$ represents the fault signal and $G$ is a known matrix for the fault term. For a non-Gaussian SDC system, a fault detection observer needs to be constructed with a selected threshold. This is different from handling Gaussian systems where a fault can be detected directly from the output data by setting a reasonable threshold without using an observer. There are various ways to detect faults in a SDC system. For the soft-bound output control system, we propose the following three fault detection methods following criteria from the initial soft-bound output control problem and the transformed PDF tracking problem.

Method A - output probability fault detection: For the softbound output control problem in (10), a fault might occur if the expected probability level of $P_{0}$ is not achieved, i.e., a fault is detected when

$$
P(k)=\int_{a_{0}}^{b_{0}} \gamma(y, u(k)) d y<P_{0} .
$$

In practice, the fault alarm interval, $\left[a_{0}^{\prime}, b_{0}^{\prime}\right]$, can be set to be wider than the soft-bound control interval, $\left[a_{0}, b_{0}\right]$, where $a_{0}^{\prime} \leq$ $a_{0}$ and $b_{0}^{\prime} \geq b_{0}$. This is equivalent to introducing additional dead-band to the fault detection within $\left[a_{0}, b_{0}\right]$, i.e., a fault is detected when

$$
P(k)=\int_{a_{0}}^{b_{0}} \gamma(y, u(k)) d y<P_{0}-\alpha_{A} .
$$

where $\alpha_{A}$ is the dead-band width that can be tuned in fault detection. This method is called 'output probability fault detection (Method A)'.

Method B - PDF tracking error fault detection: In Section II, the soft-bound output control is transformed into PDF tracking control with constrained errors, therefore the fault can be detected by checking whether the PDF tracking error moves beyond the constraint, that is, a fault is detected when

$$
\int_{a_{0}}^{b_{0}}\left(\sqrt{\gamma(y, u(k))}-\sqrt{\gamma_{g}(y)}\right)^{2} d y>\alpha_{B},
$$

where $\alpha_{B}=\max \left\{\|\Phi\| \theta_{1}^{2}\left(\alpha_{0}\right),\|\Phi\| \theta_{2}^{2}\left(\alpha_{0}\right)\right\} . \alpha_{B}$ is in fact the upper bound for $\int_{a_{0}}^{b_{0}}\left(\sqrt{\gamma(y, u(k))}-\sqrt{\gamma_{g}(y)}\right)^{2} d y$ for the soft bound PDF tracking performance. Here we use $\alpha_{B}$ for fault detection since it is directly linked to the tracking error constraint within the soft-bound region. Similar to fault detection Method A, by taking into account the dead-band effect, $\alpha_{B}=\max \left\{\|\Phi\| \theta_{1}^{2}\left(\alpha_{N}\right),\|\Phi\| \theta_{2}^{2}\left(\alpha_{N}\right)\right\}$ with $\alpha_{N}=\alpha_{0}+\alpha_{A}$.

This method is called 'PDF tracking error fault detection (Method B)'.

Method C - control performance assessment fault detection): In addition to the above two methods, we can also use the index of tracking control performance assessment (CPA) as a fault detection measure. One such index is presented as follows,

$$
\eta=\frac{S_{2}}{S_{1}+S_{2}}
$$

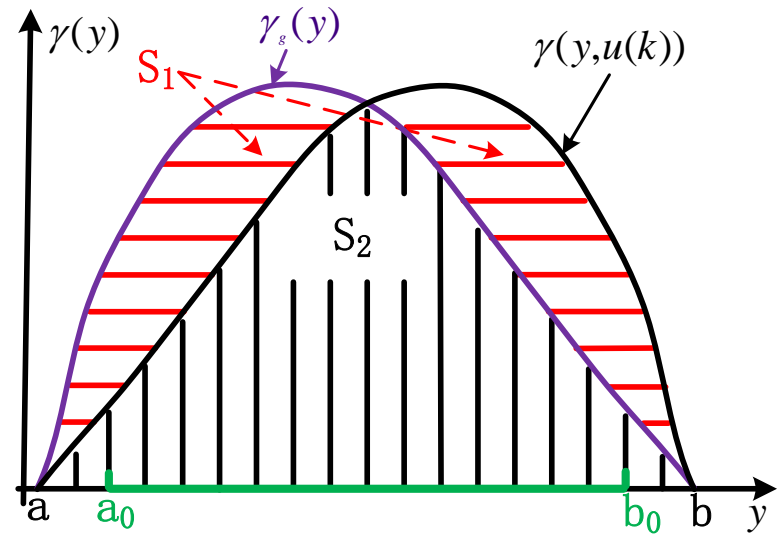

Fig. 1. Illustration of tracking control performance assessment

$S_{1}$ and $S_{2}$ are depicted in Fig. 1, in which

$$
\begin{aligned}
S_{2} & =\int_{a}^{b}\left(\gamma(y, u(k)) \cap \gamma_{g}(y)\right) d y \\
S_{1}+S_{2} & =\int_{a}^{b}\left(\gamma(y, u(k)) \cup \gamma_{g}(y)\right) d y .
\end{aligned}
$$

Here $S_{1}+S_{2}=2-S_{2}$. This performance index is a scalar taking values between 0 and $1: \eta=1$ when the process output PDF matches the target PDF completely; $\eta=0$ when there's no overlap at all between these two PDFs. A fault can therefore be detected by $\eta<\alpha_{C}$, where $\alpha_{C}$ is the fault detection threshold that can be adjusted.

To determine a proper level of $\alpha_{C}$, it is critical to compute $S_{2}$. From the illustration in Fig.1, it can be seen that

$$
S_{1}=\int_{a}^{b}\left|\gamma(y, u(k))-\gamma_{g}(y)\right| d y
$$

Furthermore, we have $S_{2}=1-\frac{1}{2} S_{1}$, and

$\frac{1}{2} S_{1}=\int_{a}^{b}\left(\gamma_{g}(y)-\gamma(y, u(k))\right) d y$, for all $\gamma_{g}(y) \geq \gamma(y, u(k))$

. From the proof of Theorem 1, it is easy to find that

$$
\begin{aligned}
\frac{1}{2} S_{1} \leq & -\min \left\{\theta_{1}^{2}\left(\alpha_{N}\right), \theta_{2}^{2}\left(\alpha_{N}\right)\right\}\|\Phi\| \\
& +2\left\|V_{g}\right\| \min \left\{\theta_{1}\left(\alpha_{N}\right), \theta_{2}\left(\alpha_{N}\right)\right\}\|\Phi\| .
\end{aligned}
$$

This fault detection method is called 'CPA fault detection (Method C)'.

Remark 5: Here three fault detection methods are proposed using different detection criteria. While Method A is based on the output PDF information, Methods B and C are developed on PDF tracking performances. In these algorithms, the output PDF is required, which can be obtained either by measurement or via a kernel density function estimation method. These options provide a wider choice of fault detection methods for non-Gaussian systems. The computational loads for these methods are similar to those conventional output PDF control problems. 


\section{B. Integrated Design for Fault Estimation and Robust Fault-} Tolerant Tracking Control

In Section III, a PI-structured controller is proposed for the soft-bound PDF tracking problem without considering possible faults in the dynamic system. This structured controller (in (28)), will be expanded for robust FTC of soft-bound PDF tracking of faulty systems, where the fault can be estimated from the output PDFs using a fault diagnosis filter as in [44], [45].

The following double-PI structure (PI controller and PI fault estimator) is constructed for the soft-bound fault-tolerant PDF tracking control.

$$
\begin{aligned}
e(k+1) & =A_{0} e(k)+B_{0} \tilde{U}(k)+E_{0} w(k)+G \tilde{F}(k) \\
\tilde{F}(k+1) & =\tilde{F}(k)-K_{p}(\varepsilon(k)-\varepsilon(k-1))-K_{I} \varepsilon(k) \\
\nu(k+1) & =\nu(k)+T_{0} \varepsilon(k) \\
\tilde{U}(k) & =K_{P_{0}} \varepsilon(k)+K_{I_{0}} \nu(k) \\
\varepsilon(k) & =\int_{a}^{b}\left(\sqrt{\gamma(y, \tilde{U}(k))}-\sqrt{\gamma_{g}(y)}\right) d y
\end{aligned}
$$

where

$$
\begin{gathered}
B_{0} \tilde{U}(k)=\left(A_{0}-I\right) V_{g}+B_{0} u(k)+\hat{F}(k), \\
\tilde{F}(k)=F(k)-\hat{F}(k), \\
\hat{F}(k+1)=\hat{F}(k)+K_{p}(\varepsilon(k)-\varepsilon(k-1))+K_{I} \varepsilon(k) .
\end{gathered}
$$

Denote $x(k)=\left[e^{\mathrm{T}}(k), \nu^{\mathrm{T}}(k), \tilde{F}^{\mathrm{T}}(k)\right]^{\mathrm{T}}$, the following state-space model is established

$$
\begin{aligned}
x(k+1)= & A_{1} x(k)+B_{1} h(k)+E w(k) \\
& +A_{2} x(k-1)+B_{2} h(k-1)
\end{aligned}
$$

where $h(k)$ is defined in (29) and

$$
\begin{aligned}
& A_{1}=\left[\begin{array}{ccc}
A_{0}+B_{0} K_{P_{0}} \Sigma_{0} & B_{0} K_{I_{0}} & G \\
T_{0} \Sigma_{0} & I & 0 \\
-\left(K_{I}+K_{P}\right) \Sigma_{0} & 0 & I
\end{array}\right], \\
& B_{1}=\left[\begin{array}{c}
B_{0} K_{P_{0}} \Sigma_{1} \\
T_{0} \Sigma_{1} \\
-\left(K_{I}+K_{P}\right) \Sigma_{1}
\end{array}\right], \quad E=\left[\begin{array}{c}
E_{0} \\
0 \\
0
\end{array}\right], \\
& B_{2}=\left[\begin{array}{c}
0 \\
0 \\
K_{P} \Sigma_{1}
\end{array}\right], A_{2}=\left[\begin{array}{ccc}
0 & 0 & 0 \\
0 & 0 & 0 \\
K_{P} \Sigma_{0} & 0 & 0
\end{array}\right] .
\end{aligned}
$$

Based on the proposed FTC structure (37), or equivalently its state-space formulation in (38), we have the following theorem.

Theorem 3: With known parameters $\lambda, \mu_{1}, \mu_{2}$ and matrix $M_{\max }$, suppose that there exist $\tilde{\Lambda}>0, S>0, K_{0}=$ $\left[K_{P_{0}}, K_{I_{0}}\right]$ and $K=\left[K_{P}, K_{I}\right]$ such that the following LMI

$$
\Psi=\left[\begin{array}{cccccc}
Q_{1} & 0 & 0 & 0 & 0 & Q_{3} \\
* & Q_{2} & 0 & 0 & 0 & A_{21}^{\mathrm{T}} R_{2} \\
* & * & -\lambda^{2} I & 0 & 0 & Q_{4} \\
* & * & * & -\lambda^{2} I & 0 & B_{21}^{\mathrm{T}} R_{2} \\
* & * & * & * & -\mu_{1}^{2} I & E^{\mathrm{T}} \tilde{\Lambda} \\
* & * & * & * & * & -\tilde{\Lambda}
\end{array}\right]<0
$$

In which,

$$
\begin{aligned}
& Q_{1}=-\tilde{\Lambda}+S+\lambda^{2} M_{\max }^{\mathrm{T}} M_{\max }+\mu_{2}^{2} \operatorname{diag}\{\Phi, 0\} \\
& Q_{2}=-S+\lambda^{2} M_{\max }^{\mathrm{T}} M_{\max } \\
& Q_{3}=A_{10}^{\mathrm{T}} \tilde{\Lambda}+A_{11}^{\mathrm{T}} R_{1}+A_{12}^{\mathrm{T}} R_{2} \\
& Q_{4}=B_{10}^{\mathrm{T}} \tilde{\Lambda}+B_{11}^{\mathrm{T}} R_{1}+B_{12}^{\mathrm{T}} R_{2}
\end{aligned}
$$

and

$$
\begin{aligned}
& A_{10}=\left[\begin{array}{ccc}
A_{0} & 0 & G \\
T_{0} \Sigma_{0} & I & 0 \\
0 & 0 & I
\end{array}\right] \quad B_{10}=\left[\begin{array}{c}
0 \\
T_{0} \Sigma_{1} \\
0
\end{array}\right] \\
& A_{11}=\left[\begin{array}{ccc}
\Sigma_{0}^{\mathrm{T}} \Sigma_{1}^{-1} & 0 & 0 \\
0 & I & 0 \\
0 & 0 & 0
\end{array}\right] \quad B_{11}=\left[\begin{array}{l}
I \\
0 \\
0
\end{array}\right] \\
& A_{12}=\left[\begin{array}{ccc}
\Sigma_{0}^{\mathrm{T}} \Sigma_{1}^{-1} & 0 & 0 \\
0 & 0 & 0 \\
0 & 0 & 0
\end{array}\right] \quad B_{12}=\left[\begin{array}{l}
I \\
0 \\
0
\end{array}\right] \\
& A_{21}=\left[\begin{array}{ccc}
0 & 0 & 0 \\
\Sigma_{0}^{\mathrm{T}} \Sigma_{1}^{-1} & 0 & 0 \\
0 & 0 & 0
\end{array}\right] \quad B_{21}=\left[\begin{array}{l}
0 \\
I \\
0
\end{array}\right] \\
& \tilde{\Lambda}=\left[\begin{array}{c}
\tilde{\Lambda}_{1} \\
\tilde{\Lambda}_{2} \\
\tilde{\Lambda}_{3}
\end{array}\right]^{\mathrm{T}} \quad R_{1}=\left[\begin{array}{c}
r_{11} \\
r_{12} \\
0
\end{array}\right] \quad R_{2}=\left[\begin{array}{c}
r_{21} \\
r_{22} \\
0
\end{array}\right]
\end{aligned}
$$

is solvable, then the closed-loop system (38) is stable and ${ }_{492}$ satisfies $e^{\mathrm{T}}(k) \Phi e(k)<\mu_{2}^{-2} \mu_{1}^{2}\|\omega(k)\|^{2}$. The corresponding $K_{0}=\left[K_{P_{0}}, K_{I_{0}}\right]$ and $K=\left[K_{P}, K_{I}\right]$ can be solved by $r_{11}=$ $\Sigma_{1} K_{P_{0}}^{\mathrm{T}} B_{0}^{\mathrm{T}} \tilde{\Lambda}_{1}, r_{21}=\Sigma_{1}\left(K_{I}+K_{p}\right)^{\mathrm{T}} \tilde{\Lambda}_{3}, r_{12}=K_{I_{0}}^{\mathrm{T}} B_{0}^{\mathrm{T}} \tilde{\Lambda}_{2}$ and $r_{22}=\Sigma_{1} K_{P}^{\mathrm{T}} \tilde{\Lambda}_{3}$

Proof: See Appendix C.

Remark 6: Different from the conventional fault estimator (either $\mathbf{P}$ - structure or I- structure), this PI- structure fault estimator has more design freedom. What's more, this integrated design for fault estimator and FTC (similar ideas see [49], [50]) with double-PI structure can be easily extended to other FTC systems.

Remark 7: The open-loop system (32) is a linear system without time-delay, but the closed-loop system in (37) is a nonlinear system that can involve time-delay terms. Therefore, the result of Theorem 3 can be easily generalized to accommodate nonlinear systems where the nonlinearity satisfies the Lipschitz conditions and/or contains a bounded time-delay term because in this integrated scheme of controller design and fault estimation, only information on output PDFs is employed.

\section{Simulation Study}

\section{A. Model and Simulation Settings}

In the following simulation study, the output PDF is defined in the range of $[a, b]=[2,7]$. The soft-bound region is set up to be $\left[a_{0}, b_{0}\right]=[4,7]$, and the soft-bound control target is specified as $\int_{4}^{7} \gamma(y, u(k)) d y \geq 0.975$, i.e., $P_{0}=0.975$. 
The output PDF is modeled by (5) with the following Bspline basis functions $(n=3, y \in[2,7])$ :

$$
\begin{aligned}
& B_{1}(y)=\frac{1}{2}(y-2)^{2} I_{1}+\left(-y^{2}+7 y-\frac{23}{2}\right) I_{2}+\frac{1}{2}(y-5)^{2} I_{3}, \\
& B_{2}(y)=\frac{1}{2}(y-3)^{2} I_{2}+\left(-y^{2}+9 y-\frac{39}{2}\right) I_{3}+\frac{1}{2}(y-6)^{2} I_{4}, \\
& B_{3}(y)=\frac{1}{2}(y-4)^{2} I_{3}+\left(-y^{2}+11 y-\frac{59}{2}\right) I_{4}+\frac{1}{2}(y-7)^{2} I_{5}
\end{aligned}
$$

where $I_{i}=\left\{\begin{array}{ll}1, & y \in[i+1, i+2] \\ 0, & \text { Otherwise }\end{array} i=1,2, \cdots 5\right.$.

With this square-root B-spline approximation, there are 2 independent weights among the 3 . It is therefore a secondorder system with the following dynamics considered

$$
V(k+1)=A_{0} V(k)+B_{0} u(k)+E_{0} \omega(k)+G F(k),
$$

where

$$
\begin{aligned}
A_{0} & =\left[\begin{array}{cc}
0.978 & 0.03 \\
0.09 & 0.975
\end{array}\right], & B_{0} & =\left[\begin{array}{ll}
0.02 & 0.01 \\
0.03 & 0.02
\end{array}\right], \\
E_{0} & =\left[\begin{array}{l}
0.02 \\
0.04
\end{array}\right], & G & =\left[\begin{array}{l}
0.01 \\
0.02
\end{array}\right] .
\end{aligned}
$$

The disturbance term, $\omega(k)$, is chosen as a stochastic variable following the uniform distribution defined within the range of $[-0.1,0.1]$.

The time-varying fault term is constructed as follows

$$
F(k)= \begin{cases}0, & 0<k \leq 80 \\ 0.04(k-80), & 80<k \leq 130 \\ 2-0.04(k-130), & 130<k \leq 180 \\ 0, & 180<k \leq 230 \\ 1.2, & k>230\end{cases}
$$

In the first stage of the process when $k \leq 80$, it is assumed that the system is fault free.

In the following simulation study, three target PDFs, $\gamma_{g 1}(y)$, $\gamma_{g 2}(y)$ and $\gamma_{g 3}(y)$, are selected to investigate how to tune the algorithm to achieve effective and robust performance. These target PDFs are also modeled by the same B-spline approximation using $B_{1}, B_{2}$ and $B_{3}$. Their corresponding weights vectors, values of $P_{1}=\int_{4}^{7} \gamma_{g}(y) d y$, and the probability discrepancy factor, $\alpha_{0}=P_{1}-P_{0}$, are listed in Table I. The three target PDF curves are shown in Fig. 2.

TABLE I

THREE SELECTED TARGET PDFS AND RELEVANT PARAMETERS

\begin{tabular}{c|c|c|c}
\hline \hline & $V_{g}$ & $P_{1}$ & $\alpha_{0}$ \\
\hline target PDF1 & {$\left[\begin{array}{ll}0.152, & 0.204\end{array}\right]^{\mathrm{T}}$} & 0.9796 & 0.0046 \\
\hline target PDF2 & {$\left[\begin{array}{ll}0.080,0.435\end{array}\right]^{\mathrm{T}}$} & 0.9796 & 0.0046 \\
\hline target PDF3 & {$\left[\begin{array}{ll}0.010,0.240\end{array}\right]^{\mathrm{T}}$} & 0.9965 & 0.0215 \\
\hline \hline
\end{tabular}

In all simulations, 20 times Monte Carlo computations are implemented, and the initial weights vector is always set to be $V_{0}=[0.5,0.3]^{\mathrm{T}}$.

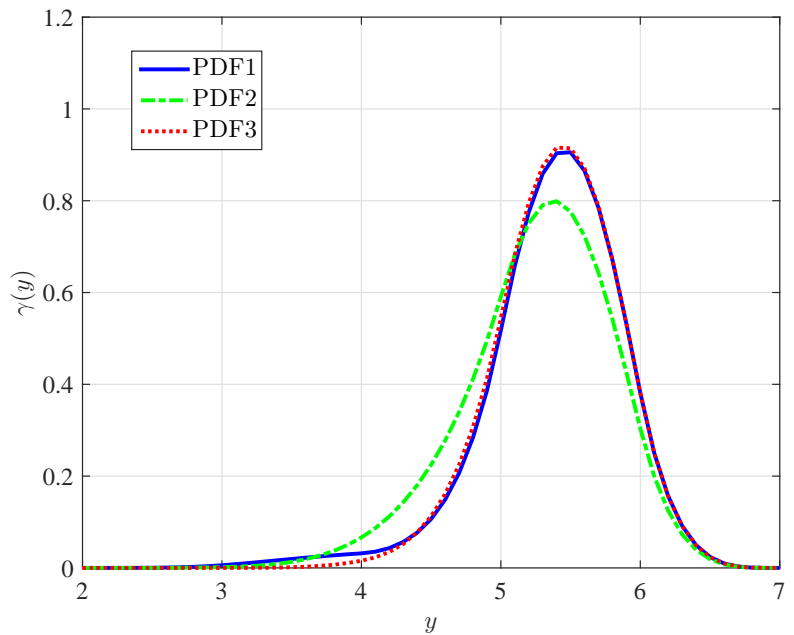

Fig. 2. Three target PDFs. Mean value of PDF 1, 2, 3: $5.3738,5.2609,5.4022$, central value of $[4,7]$ is 5.5

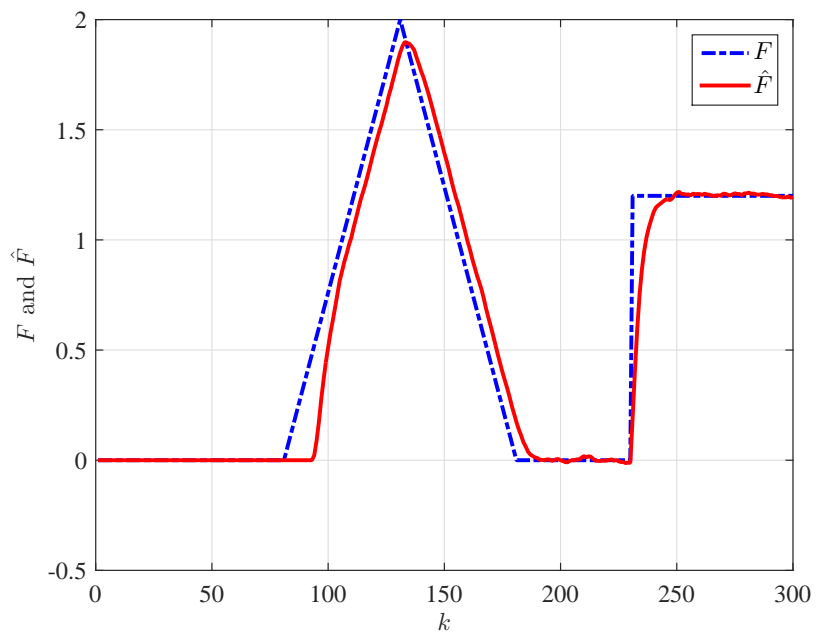

Fig. 3. Fault and fault estimation signals over time

\section{B. Fault Detection and FTC for Target PDF1}

With target PDF1, the transformed PDF tracking problem has error constraint of $\alpha_{2}=\alpha_{1} \leq 6.2384 \times 10^{-4}\left(M_{\min }=\right.$ $\left.0.3315, M_{\max }=2.2103, \theta_{1}=0.0125, \theta_{2}=0.0119\right)$. The profiles of the fault signal and its estimation are illustrated in Fig. 3, from which a rapid response and a small estimation error can be observed after the fault is detected.

In the fault-free case $(k \leq 80)$, the parameters of the LMI method are $\lambda^{2}=0.01, \mu_{1}^{2}=0.04$ and $\mu_{2}^{2}=1.0$. The structured fault-free controller in (28) is applied and the control gain matrix $K_{0}$ is obtained from (27) to be

$$
K_{0}=\left[K_{P_{0}}, K_{I_{0}}\right]=\left[\begin{array}{ll}
-0.4330 & -0.6216 \\
-5.0970 & -6.5786
\end{array}\right] .
$$

When applying FTC based on the detected fault, the parameters of the LMI method are selected as $\lambda^{2}=0.015, \mu_{1}^{2}=0.04$, $\mu_{2}^{2}=1.0$, and $\alpha_{A}=0.001$. The double-PI structured FTC in (37) is applied, and the control input gain matrix and the vector 


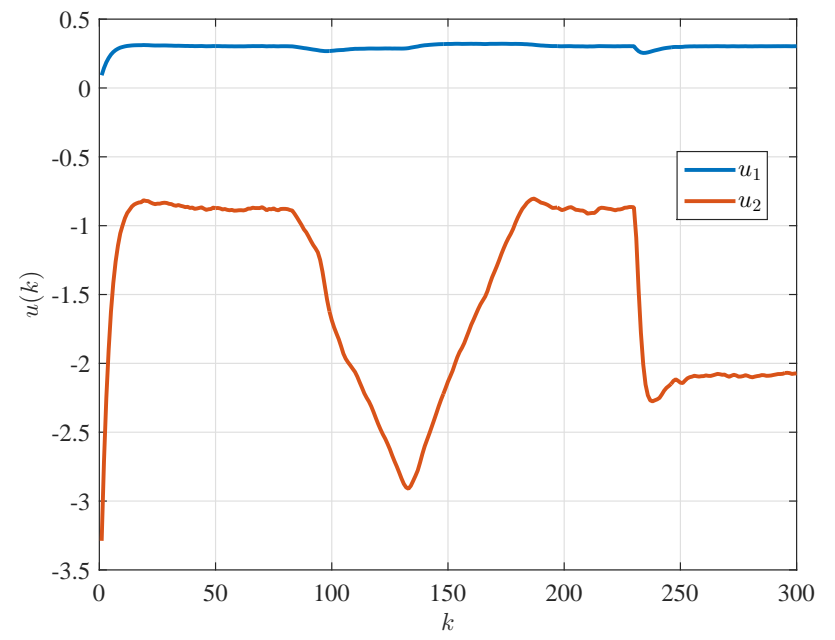

Fig. 4. Time profiles of the two FTC input signals (target PDF1)

of fault estimation parameters are calculated to be

$$
\begin{aligned}
K_{0} & =\left[K_{P_{0}}, K_{I_{0}}\right]=\left[\begin{array}{ll}
-0.4272 & -0.6318 \\
-6.9787 & -3.8001
\end{array}\right], \\
K & =\left[K_{P}, K_{I}\right]=[-3.8936,5.1032] .
\end{aligned}
$$

The three fault detection methods are simulated based on which the proposed FTC is developed. The results from 20 Monte Carlo simulations are averaged and shown in Figs. 4 - 7. Figure 4 displays the time profiles of the two control signals. Under the proposed soft-bound control strategy, the output variable falls within the specified region of $[4,7]$ with a probability around $98 \%$ in the fault-free condition (see Fig. 5 for the period up to $k=80$ ). When a fault occurs in the system after the 80th sample time, the robust fault tolerant tracking control is activated once the fault is detected (here fault detection Method A is used in Fig. 4).

Fig. 5 shows the FTC result using the output probability for fault detection (Method A); Fig. 6 illustrates the FTC result with fault detection made on the PDF tracking error (Method B); and Fig. 7 presents the results using the CPA index in fault detection (Method C). In these three figures, the dash-dot lines are the fault detection threshold lines. The fault detection criteria parameters are: $\alpha_{A}=0.001$, i.e. $P_{0}-\alpha_{A}=0.974$, for Method A; $\alpha_{B}=1.1 \times 10^{-3}$ for Method B; and $\alpha_{C}=0.9556$ for Method C. These results demonstrate that all three fault detection methods can be used to detect faults effectively when $P_{1}$ is close to $P_{0}$. Satisfactory control performance has been achieved using the proposed soft-bound output PDF controller.

\section{Comparison of Fault Detection Time using Target PDF1}

The fault detection time using the three different methods are compared in Table II for target PDF1, where ' $\mathrm{C} 1$ ' represents $\alpha_{A}=0.001$ and ' $\mathrm{C} 2$ ' represents $\alpha_{A}=0.005$. It can be seen that it takes certain amount of time to detect the fault for a dynamic system $(10-14$ samples in all of the 20 Monte Carlo simulations for this example). Among the three methods, the fault detection time using Method A is the shortest. This is because the fault detection threshold used in Method A is directly linked to the soft-bound output control

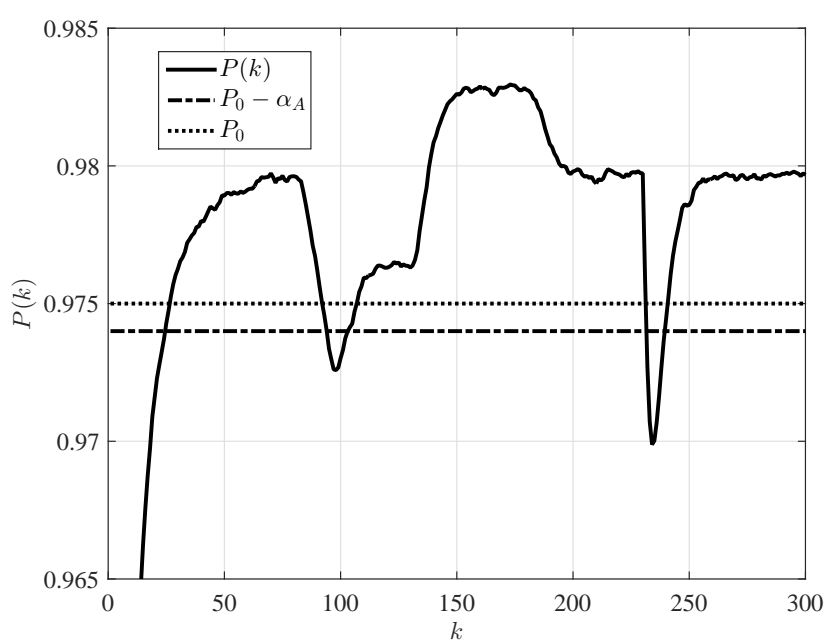

Fig. 5. FTC with fault detection Method A based on output probability (target PDF1, $\left.\alpha_{A}=0.001\right)$

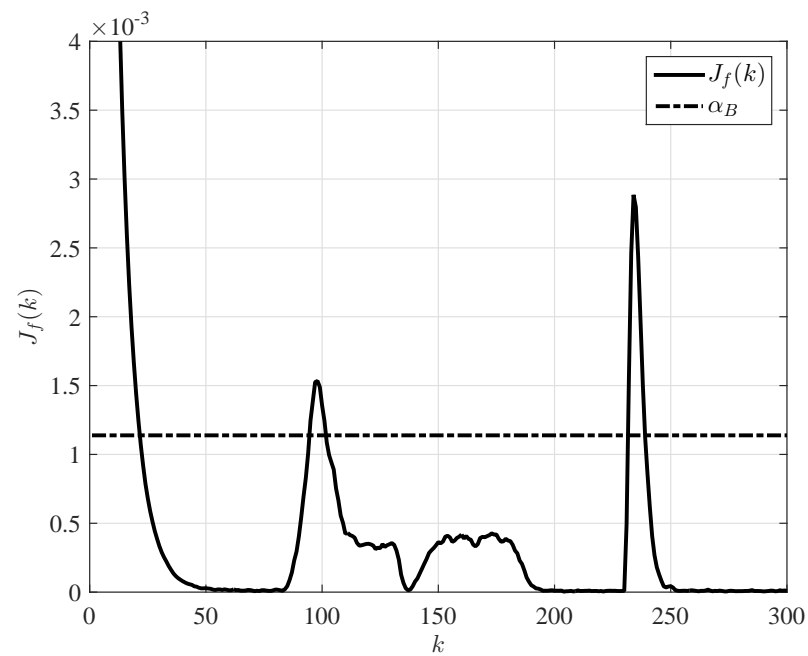

Fig. 6. FTC with fault detection Method B based on PDF tracking error (target PDF1, $\alpha_{B}=1.1 \times 10^{-3}$ )

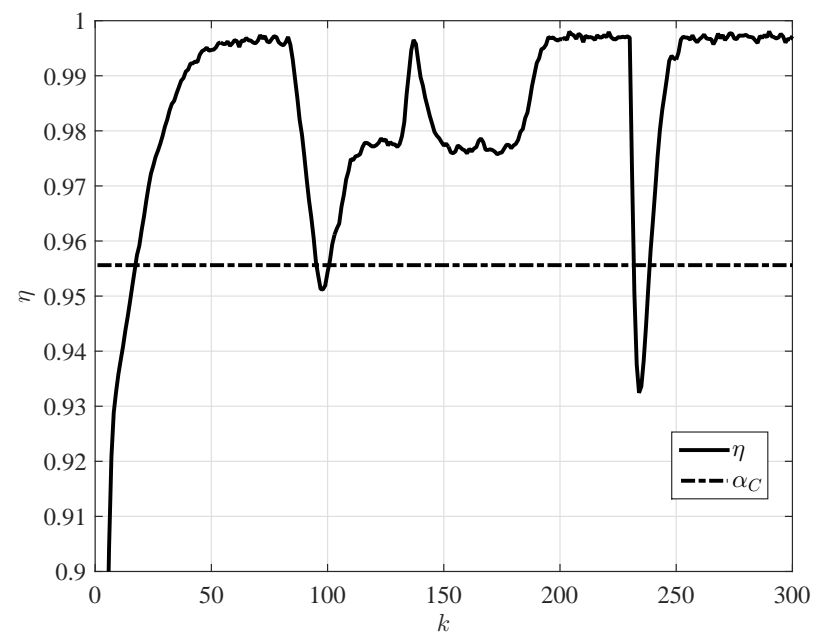

Fig. 7. FTC with fault detection Method $\mathrm{C}$ based on tracking CPA (target PDF1, $\left.\alpha_{C}=0.9556\right)$ 
goal. Methods B and C, however, take thresholds following the transformed PDF tracking control with constrained errors, which are slightly more conservative and therefore take longer time for fault detection.

TABLE II

FAULT DETECTION TIME OF THE THREE DETECTION METHODS (TARGET PDF1)

\begin{tabular}{r|r|r|r|r|r|r}
\hline \multirow{2}{*}{} & \multicolumn{2}{|c|}{ Method A } & \multicolumn{2}{c|}{ Method B } & \multicolumn{2}{c}{ Method C } \\
\cline { 2 - 7 } & $\mathrm{C} 1$ & $\mathrm{C} 2$ & $\mathrm{C} 1$ & $\mathrm{C} 2$ & $\mathrm{C} 1$ & $\mathrm{C} 2$ \\
\hline 1 & 94 & 100 & 95 & 103 & 95 & 103 \\
\hline 2 & 94 & 101 & 95 & 103 & 95 & 103 \\
\hline 3 & 94 & 100 & 96 & 102 & 96 & 102 \\
\hline 4 & 95 & 102 & 95 & 103 & 97 & 103 \\
\hline 5 & 93 & 101 & 94 & 103 & 94 & 103 \\
\hline 6 & 97 & 100 & 97 & 104 & 97 & 104 \\
\hline 7 & 96 & 102 & 97 & 103 & 97 & 103 \\
\hline 8 & 94 & 99 & 95 & 104 & 95 & 104 \\
\hline 9 & 94 & 100 & 95 & 105 & 95 & 105 \\
\hline 10 & 95 & 100 & 95 & 102 & 96 & 102 \\
\hline 11 & 96 & 101 & 96 & 103 & 97 & 103 \\
\hline 12 & 95 & 102 & 96 & 103 & 96 & 104 \\
\hline 13 & 94 & 100 & 94 & 104 & 95 & 104 \\
\hline 14 & 93 & 101 & 96 & 102 & 96 & 102 \\
\hline 15 & 93 & 102 & 94 & 104 & 95 & 104 \\
\hline 16 & 96 & 100 & 96 & 104 & 96 & 104 \\
\hline 17 & 94 & 101 & 97 & 103 & 97 & 103 \\
\hline 18 & 93 & 100 & 94 & 102 & 95 & 102 \\
\hline 19 & 95 & 100 & 95 & 102 & 95 & 102 \\
\hline 20 & 92 & 101 & 96 & 102 & 97 & 102 \\
\hline Mean & 94.35 & 100.65 & 95.4 & 103.05 & 95.8 & 103.1 \\
\hline \hline
\end{tabular}

\section{Fault Detection and FTC for Target PDF2}

Target PDF2 is selected to have the same level of $P_{1}$ as target PDF1, and therefore share the same probability discrepancy factor, $\alpha_{0}=0.0046$. However, the shape of target PDF2 is different from target PDF1, which are defined by $V_{g 1}$ and $V_{g 2}$, respectively. Therefore, their corresponding tracking error constraint bounds are different. For target PDF2, $\alpha_{2}=\alpha_{1} \leq 2.1399 \times 10^{-4}$, while for target PDF1, $\alpha_{2}=\alpha_{1} \leq 6.2384 \times 10^{-4}$ ), when $\alpha_{A}=0.001$.

The PDF tracking error constraint is smaller for target PDF2 compared to target PDF1. We need to select smaller parameters to meet the tracking error constraint requirements. In this case, $\lambda^{2}=0.01, \mu_{1}^{2}=0.02, \mu_{2}^{2}=1.0$, and The doublePI structured FTC in (37) is again applied. The control input gain matrix and the vector of fault estimation parameters are calculated to be

$$
\begin{aligned}
K_{0} & =\left[K_{P_{0}}, K_{I_{0}}\right]=\left[\begin{array}{ll}
-0.4330 & -0.6216 \\
-5.0976 & -6.5771
\end{array}\right], \\
K & =\left[K_{P}, K_{I}\right]=[-3.7832,4.9012] .
\end{aligned}
$$

Note with smaller parameters in $\left(\mu_{1}, \mu_{2}^{-1}\right)$, there is a larger numerical risk of getting no solution to the LMI. For this reason, in choosing a target PDF for the transformed PDF tracking control, the one with a larger value of error constraint is favored when appropriate.

Figs. 9 - 11 present the FTC results under target PDF2 using three different fault detection methods. The fault detection criteria parameters are: $\alpha_{A}=0.001$ (Method A), $\alpha_{B}=3.6286 \times 10^{-4}($ Method B $), \alpha_{C}=0.9461($ Method C)

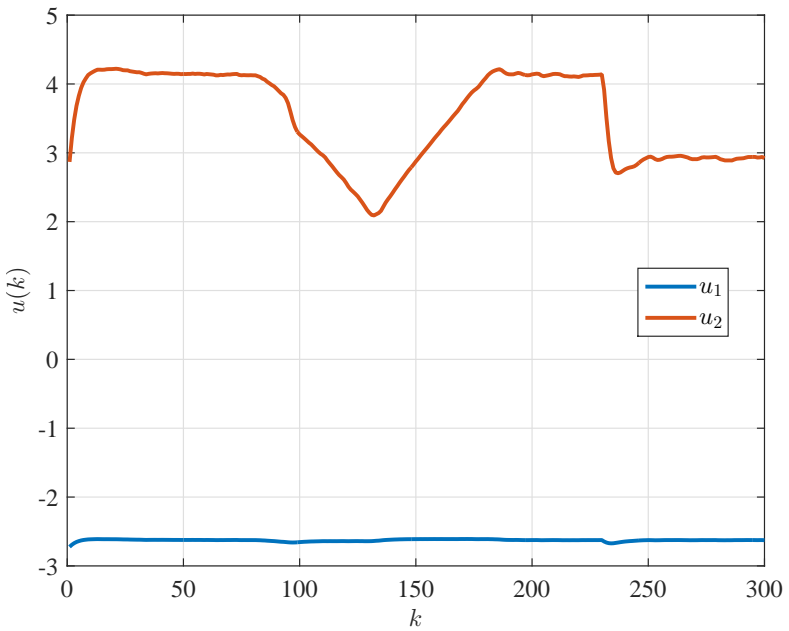

Fig. 8. Time profiles of the two FTC input signals (target PDF2)

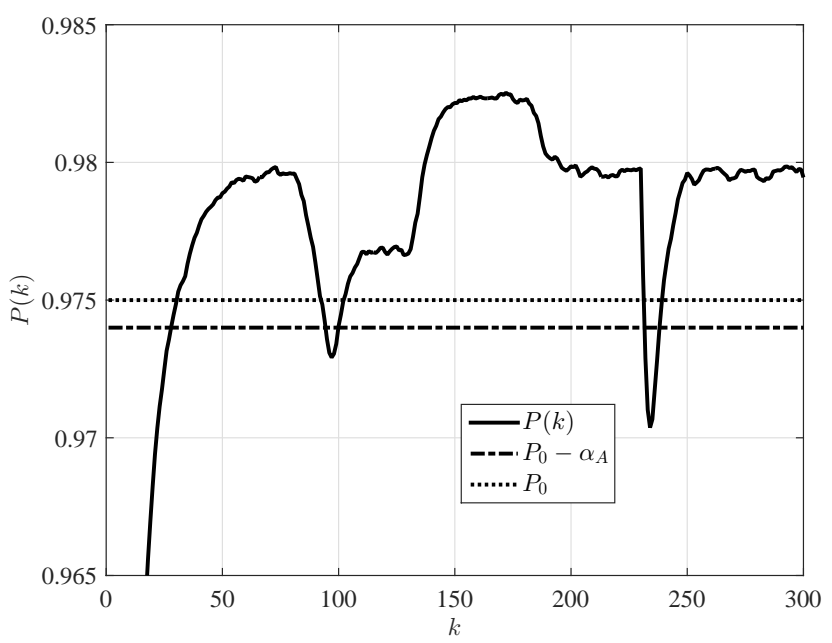

Fig. 9. FTC with fault detection Method A based on output probability (target PDF2, $\left.\alpha_{A}=0.001\right)$

The two control signals are shown in Fig. 8 for target PDF2. Comparing the results for using target PDF1 and target PDF2, it can be seen that their FTC performances are very similar, however, the control cost with target PDF2 is much higher than that using target PDF1. This suggests that the selection of the target PDF will affect the controller design. Even with the same level of $P_{1}$, two target PDFs in different shapes will lead to different results.

\section{Fault Detection and FTC for Target PDF3}

Target PDF3 is selected to have a larger value of $P_{1}$ compared with target PDF1 \& 2 . The difference between $P_{1}$ and $P_{0}$ is thus increased (see $\alpha_{0}=0.0215$ in Table I). In this case, the error constraints of the transformed PDF tracking problem are $\alpha_{2}=\alpha_{1} \leq 0.0128$ with $\alpha_{A}=0.001$. Setting $\lambda^{2}=0.02, \mu_{1}^{2}=0.36, \mu_{2}^{2}=1.0$, the control input gain matrix and the vector of fault estimation parameters are

$$
\begin{aligned}
K_{0} & =\left[K_{P_{0}}, K_{I_{0}}\right]=\left[\begin{array}{ll}
-0.4274 & -0.6319 \\
-6.8495 & -3.7212
\end{array}\right], \\
K & =\left[K_{P}, K_{I}\right]=[-3.5738,5.2102] .
\end{aligned}
$$




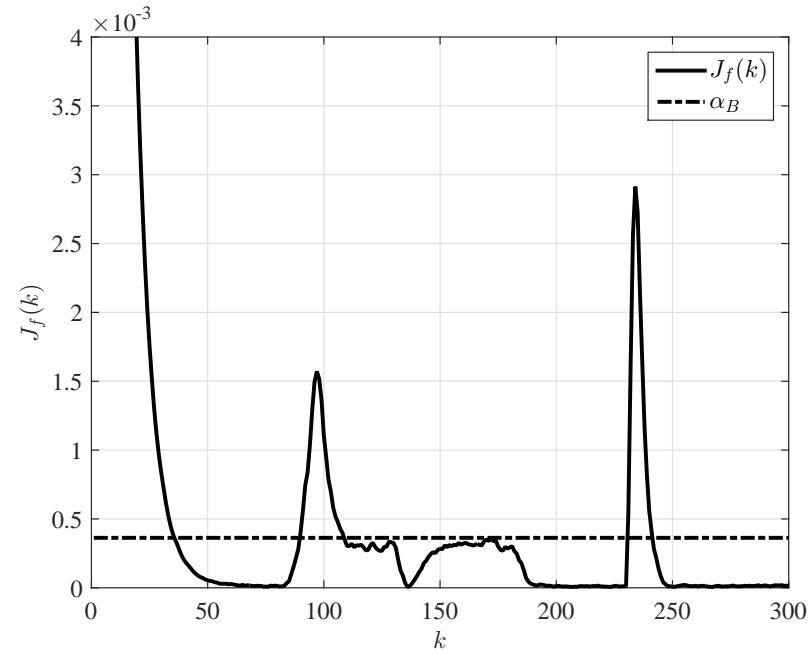

Fig. 10. FTC with fault detection Method $\mathrm{B}$ based on PDF tracking error (target PDF2, $\alpha_{B}=3.6286 \times 10^{-4}$ )

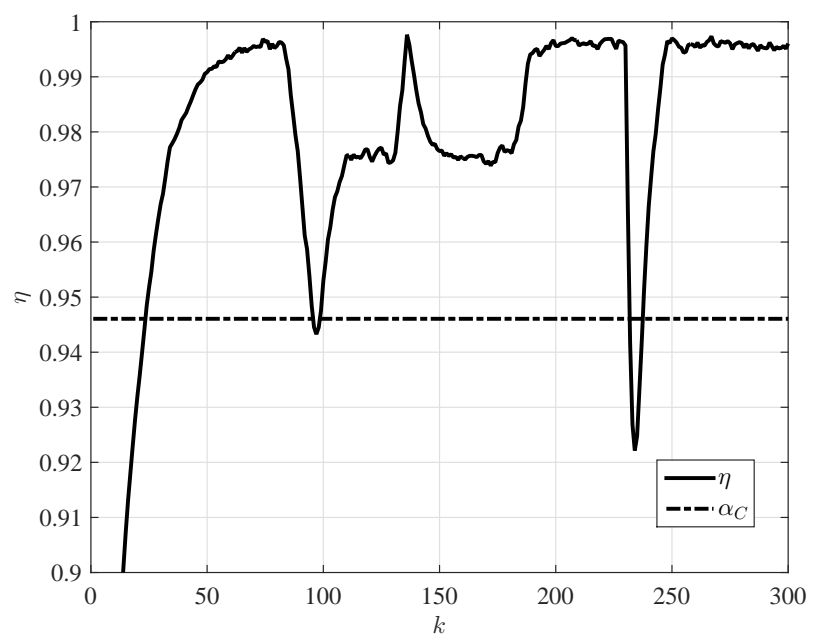

Fig. 11. FTC with fault detection Method $\mathrm{C}$ based on tracking CPA (target PDF2, $\left.\alpha_{C}=0.9461\right)$

The fault detection and FTC simulation results are illustrated in Figs. 12 - 13. Here only the fault detection Method A is used for comparison.

Comparing the results from target PDF3 to those with target PDF1 \& 2, it can be argued that the fault detection is more difficult when using target PDF3 because the difference between $P_{1}$ and $P_{0}$ is larger. From the 20 Monte-Carlo simulations, the averaged fault detection time (point) using Method A is 129.65 for target PDF3, 94.15 for target PDF2, and 94.35 for target PDF1. From the robust control point of view, a better robustness is achieved for target PDF3 although the cost is larger control activities.

\section{E. Comparison of Control W/O Fault Tolerant Design}

We then applied the structured fault-free controller in (28) to the same SDC system for comparison with the proposed controller in (37). Target PDF1 \& 3 are selected for comparison study with and without FTC design.

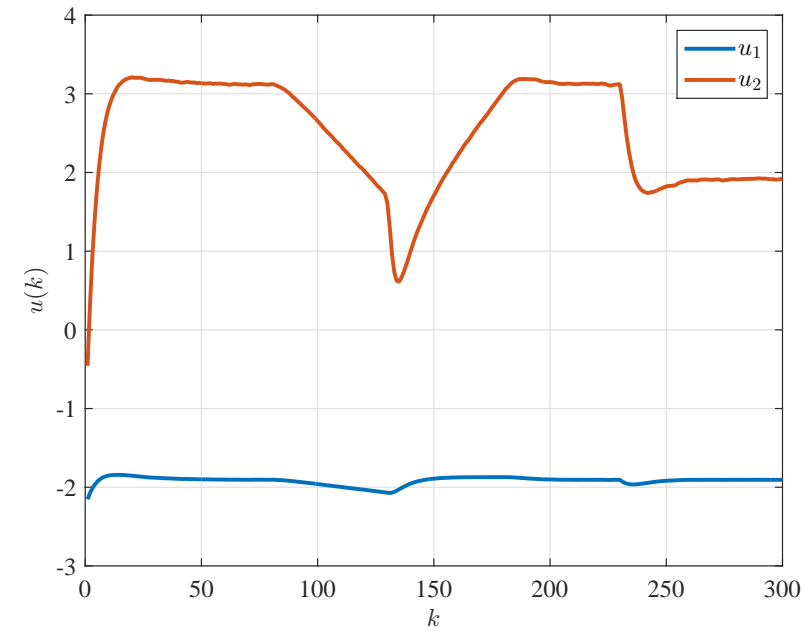

Fig. 12. Time profiles of the two FTC input signals (target PDF3, $\alpha_{A}=$ $0.001)$

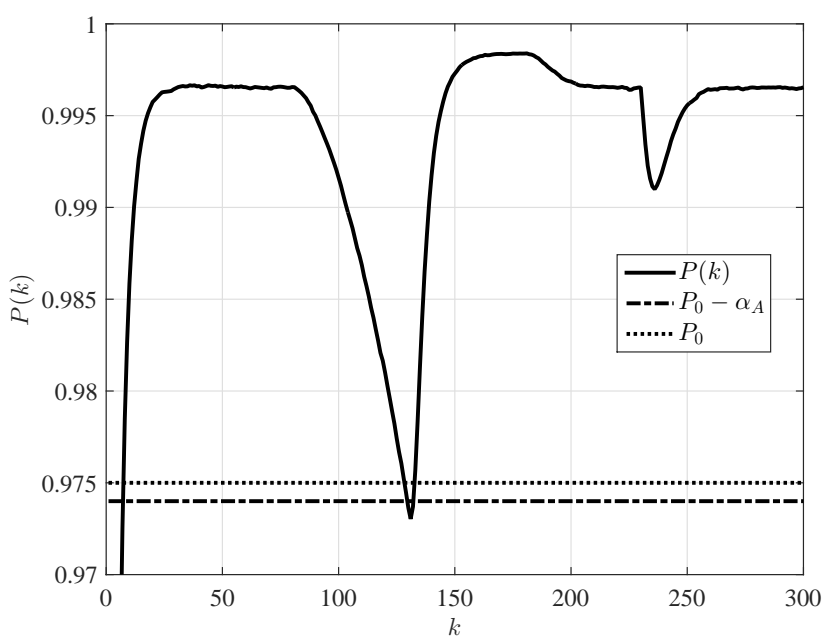

Fig. 13. FTC with fault detection Method A based on output probability (target PDF3, $\alpha_{A}=0.001$ )

Figs. 14 and 15 illustrate the soft-bound output control results for target PDF1 and target PDF3, respectively. Compared with the corresponding results under the proposed FTC, see Fig. 5 for target PDF1 and Fig. 13 for target PDF3, it can be seen that the control performance without FTC is rather poor when the system is in presence of faults. This surely indicates the importance, and also the effectiveness, of using the proposed FTC for a faulty SDC system. The control signals from the fault-free design are shown in Figs. 16 and Fig. 17 for target PDF $1 \& 3$, respectively, from which it can be seen that the control cost for target PDF3 is higher than that of target PDF1. This is a consistent conclusion obtained for using FTC.

From the above extensive simulation studies, it can be concluded that the proposed integrated fault detection and FTC design can achieve satisfactory control performance for the soft-bound output control problem. The selection of the probability discrepancy factor, $\alpha_{0}$, is crucial to controller design. The larger is $\alpha_{0}$, the better FTC robustness can be obtained but with a price of larger control activities. The 


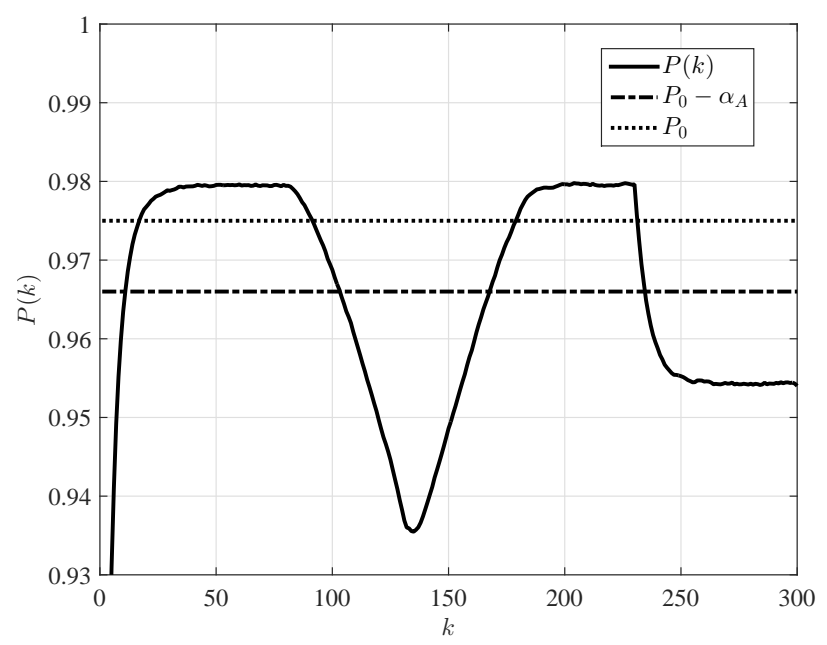

Fig. 14. Output probability without FTC (target PDF1)

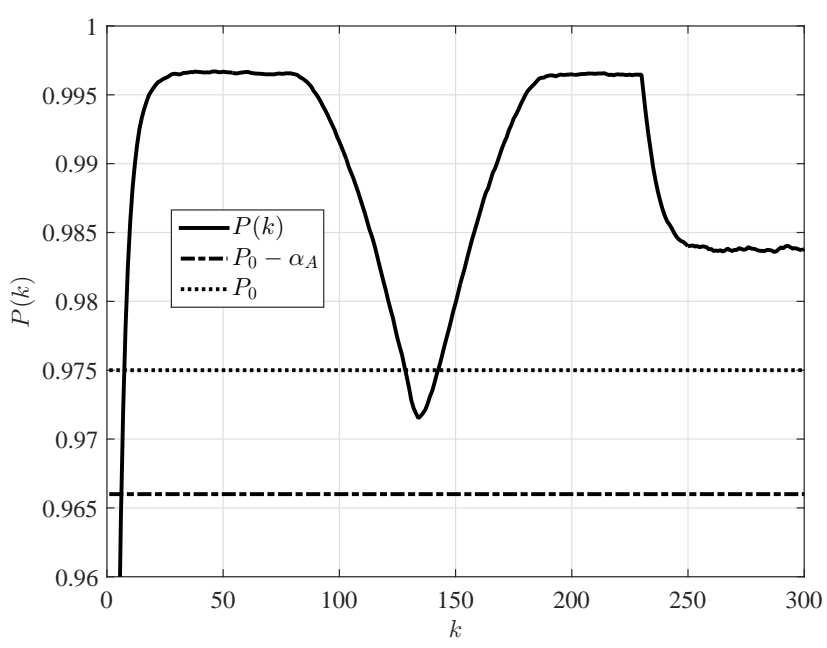

Fig. 15. Output probability without FTC (target PDF3)

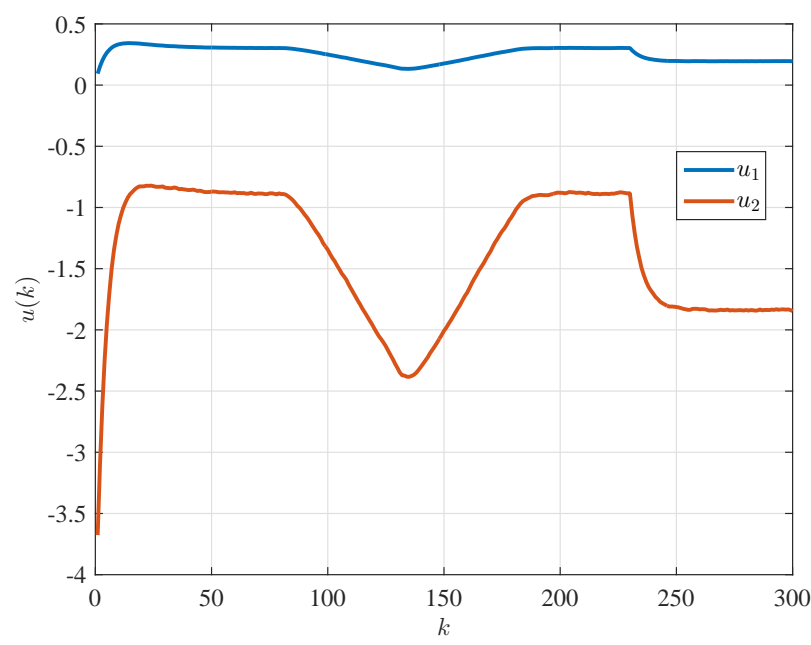

Fig. 16. Time profiles of the two FTC input signals (target PDF1)

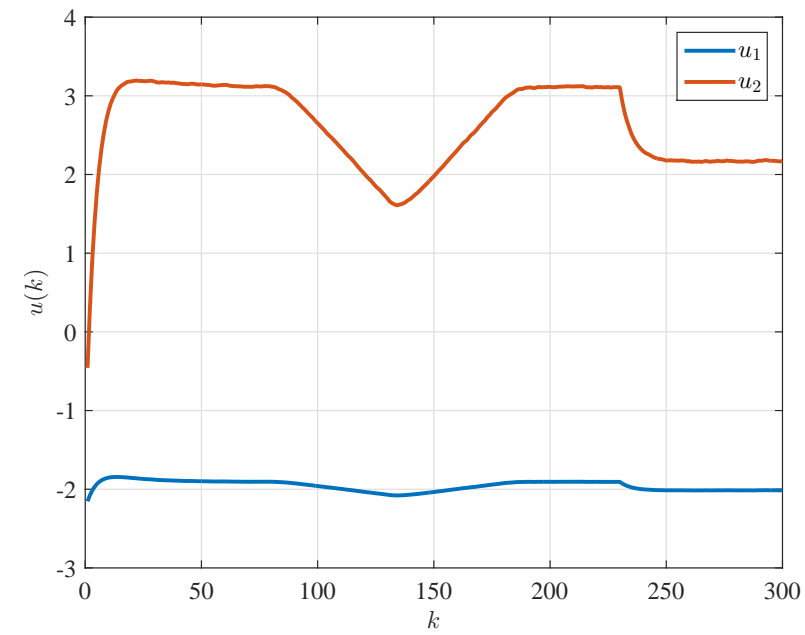

Fig. 17. Time profiles of the two FTC input signals (target PDF 3)

selection of target PDF will also affect the controller design, for example, under the same level of $\alpha_{0}$, the target PDF corresponding to larger PDF tracking error constraint will be more suitable for numerical searching of the control solution through LMI.

\section{CONCLUSIONS}

In this paper, a fault-tolerant soft-bound interval control problem has been discussed for general non-Gaussian SDC systems. The aim is to control the output variable within the required interval at a certain (large) probability level. This idea is inspired by real process control requirements, e.g. product quality, operational cost, etc., to be achieved under stochastic environments, where it is unrealistic to set up hard-bound constraints. To achieve the overall objective of developing robust FTC for soft-bound interval control systems, our work are conducted including the following four major parts: (I) propose and formulate the soft-bound interval control problem and recast it into output PDF tracking problem with an added constraint on tracking errors; (II) develop various fault detection methods following the initial soft-bound interval control problem and the transformed PDF tracking problem, and (III) develop the integrated fault estimation and FTC with double PI-structured design. The proposed algorithm has been simulated under various scenarios and satisfactory control performances have been achieved in presence of time-varying faults.

The overall robustness performance of the proposed control strategy can be achieved from various ways within the softbound design framework, among them the following are perhaps most relevant. Firstly, compared with hard-bound control, the robustness of soft-bound control can be obtained by setting up the probability level, $P_{0}$. In general, a smaller value of $P_{0}$ would lead to a less conservative controller. Similarly, the robustness effects can be obtained by tuning the soft-bound control interval, $\left[a_{0}, b_{0}\right]$. The wider is this region, the less conservative is the controller. Secondly, the robustness can be obtained from FTC design in the sense that the system is able to handle time-varying faults. We've also included an 
uncertainty term in the model as a common practice in robust controller design. Thirdly, the PI-structured integration design for both fault estimation and FTC provides robustness to some extent as widely accepted by control practice.

\section{APPENDIX}

\section{A. Proof of Lemma 2}

For simplicity, assume

$$
\Phi_{3} \geq V^{\mathrm{T}}\left(k_{2}\right) \Phi_{0} V\left(k_{2}\right) \geq V^{\mathrm{T}}\left(k_{1}\right) \Phi_{0} V\left(k_{1}\right),
$$

then for the two functions,

$$
\begin{gathered}
g_{1}=\sqrt{\Phi_{3}-V^{\mathrm{T}}\left(k_{1}\right) \Phi_{0} V\left(k_{1}\right)}-\sqrt{\Phi_{3}-V^{\mathrm{T}}\left(k_{2}\right) \Phi_{0} V\left(k_{2}\right)}, \\
g_{2}=\sqrt{V^{\mathrm{T}}\left(k_{2}\right) \Phi_{0} V\left(k_{2}\right)}-\sqrt{V^{\mathrm{T}}\left(k_{1}\right) \Phi_{0} V\left(k_{1}\right)},
\end{gathered}
$$

denoting

$$
\begin{aligned}
& V^{\mathrm{T}}\left(k_{1}\right) \Phi_{0} V\left(k_{1}\right)=\Phi_{3} \sin ^{2} \vartheta, \\
& V^{\mathrm{T}}\left(k_{2}\right) \Phi_{0} V\left(k_{2}\right)=\Phi_{3} \sin ^{2} \beta,
\end{aligned}
$$

respectively, where $\pi / 2 \geq \beta \geq \vartheta \geq 0$, we have

$$
\begin{aligned}
g_{1} & =\sqrt{\Phi_{3}}[\cos \vartheta-\cos \beta] \\
& =2 \sqrt{\Phi_{3}}\left[\sin \left(\frac{\vartheta+\beta}{2}\right) \sin \left(\frac{\beta-\vartheta}{2}\right)\right], \\
g_{2} & =\sqrt{\Phi_{3}}[\sin \beta-\sin \vartheta] \\
& =2 \sqrt{\Phi_{3}}\left[\sin \left(\frac{\beta-\vartheta}{2}\right) \cos \left(\frac{\vartheta+\beta}{2}\right)\right] .
\end{aligned}
$$

Therefore, if $\vartheta \neq \beta$, then $g_{1} \leq M_{1} g_{2}$ when $M_{1} \geq \tan \left(\frac{\vartheta+\beta}{2}\right)$; if $\vartheta=\beta$ then $g_{1}=M_{1} g_{2}$ for any real values of $M_{1}$. Consider the case that $M_{1} \geq \tan \left(\frac{\vartheta+\beta}{2}\right)$ together with the use of Lemma 1 , we can find $M_{\max }$

$$
M_{\max }=\frac{M_{1} \frac{\lambda_{\max }\left(\Phi_{0}\right)}{\sqrt{\lambda_{\min }\left(\Phi_{0}\right)}}+\left\|\Phi_{2}\right\|}{\left\|\Phi_{3}\right\|} .
$$

Similarly, for given $V\left(k_{1}\right)$ and $V\left(k_{2}\right)$ such that

$$
\Phi_{3} \geq V^{\mathrm{T}}\left(k_{2}\right) \Phi_{0} V\left(k_{2}\right) \geq V^{\mathrm{T}}\left(k_{1}\right) \Phi_{0} V\left(k_{1}\right),
$$

if $M_{2} \leq \cot \left(\frac{\vartheta+\beta}{2}\right)$, then

$$
\begin{aligned}
& \sqrt{\Phi_{3}-V^{\mathrm{T}}\left(k_{1}\right) \Phi_{0} V\left(k_{1}\right)}+\sqrt{\Phi_{3}-V^{\mathrm{T}}\left(k_{2}\right) \Phi_{0} V\left(k_{2}\right)} \\
\geq & M_{2}\left(\sqrt{V^{\mathrm{T}}\left(k_{2}\right) \Phi_{0} V\left(k_{2}\right)}+\sqrt{V^{\mathrm{T}}\left(k_{1}\right) \Phi_{0} V\left(k_{1}\right)}\right),
\end{aligned}
$$

and

$$
M_{\min }=\frac{\left\|M_{2} \sqrt{\lambda_{\min }\left(\Phi_{0}\right)}-\right\| \Phi_{2}\|\|}{\left\|\Phi_{3}\right\|} .
$$

However, for arbitrary $V\left(k_{1}\right)$ and $V\left(k_{2}\right)$, the value of $M_{1}$ could be infinitely large and $M_{2}$ infinitely small. This indicates that in order to find a feasible $M_{\max }$, certain constraints need to be satisfied. For example, if $V^{\mathrm{T}}\left(k_{1}\right) \Phi_{0} V\left(k_{1}\right)+$ $V^{\mathrm{T}}\left(k_{2}\right) \Phi_{0} V\left(k_{2}\right) \leq \Phi_{3}$ or $\vartheta+\beta \leq \pi / 2$, then the maximum value of $M_{1}$ and the minimum value of $M_{2}$ are both 1 .

\section{B. Proof of Theorem 1}

Assume $\int_{a_{0}}^{b_{0}} \gamma(y, u(k)) d y \leq P_{1}$, then we have

$\int_{a_{0}}^{b_{0}}\left(\gamma_{g}(y)-\gamma(y, u(k))\right) d y \leq \alpha_{0}$

$\Leftrightarrow \int_{a_{0}}^{b_{0}}\left[\sqrt{\gamma_{g}(y)}-\sqrt{\gamma(y, u(k))}\right]$

$\times\left[\sqrt{\gamma_{g}(y)}+\sqrt{\gamma(y, u(k))}\right] d y \leq \alpha_{0}$

$\Leftrightarrow e_{g}^{\mathrm{T}} \Phi_{01}\left(V(k)+V_{g}\right)+e_{g}^{\mathrm{T}} \Phi_{02} H\left(V(k)+V_{g}\right)$

$+H\left(e_{g}\right) \Phi_{02}^{\mathrm{T}}\left(V(k)+V_{g}\right)+H\left(e_{g}\right) H\left(V(k)+V_{g}\right) \Phi_{03}$

$\leq \alpha_{0}$

where $e_{g}=V(k)-V_{g}, H\left(e_{g}\right)=H(V(k))-H\left(V_{g}\right)$, and $H\left(V(k)+V_{g}\right)=H\left(V_{g}\right)+H(V(k))$.

Using Lemma 2, if the following inequality

$$
-\left\|e_{g}\right\|^{2}\left\|\Phi_{\min }\right\|+2\left\|V_{g}\right\|\left\|e_{g}\right\|\left\|\Phi_{\min }\right\| \leq \alpha_{0}
$$

holds, then (44) will also hold. For the weights tracking error $e(k)=V(k)-V_{g}$, from (45), we have

$$
\begin{aligned}
\|e(k)\| & \leq \frac{\left\|V_{g}\right\|\left\|\Phi_{\min }\right\|-\sqrt{\left\|V_{g}\right\|^{2}\left\|\Phi_{\min }\right\|^{2}-\alpha_{0}\left\|\Phi_{\min }\right\|}}{\left\|\Phi_{\min }\right\|} \\
& =\theta_{1} .
\end{aligned}
$$

Similarly, for $\int_{a_{0}}^{b_{0}} \gamma(y, u(k)) d y \geq P_{1}$, we have

$$
\begin{aligned}
\|e(k)\| & \leq \frac{\sqrt{\left\|V_{g}\right\|^{2}\left\|\Phi_{\min }\right\|^{2}+\alpha_{0}\left\|\Phi_{\min }\right\|}-\left\|V_{g}\right\|\left\|\Phi_{\min }\right\|}{\left\|\Phi_{\min }\right\|} \\
& =\theta_{2} .
\end{aligned}
$$

Furthermore, for the output PDF tracking errors in the definition region and the soft-bound region, respectively, we have the following bounding

$$
\begin{aligned}
& \int_{a}^{b}\left(\sqrt{\gamma(y, u(k))}-\sqrt{\gamma_{g}(y)}\right)^{2} d y \\
& \quad \leq\left(\left\|\Phi_{1}\right\|+2\left\|M_{\max }\right\|\left\|\Phi_{2}\right\|+\left\|M_{\max }\right\|^{2}\left\|\Phi_{3}\right\|\right)\|e\|^{2} \\
& \quad=\|\Phi\|\|e\|^{2}
\end{aligned}
$$

Therefore,

$$
\alpha_{1}=\min \left\{\|\Phi\| \theta_{1}^{2},\|\Phi\| \theta_{2}^{2}\right\} .
$$

\section{Proof of Theorem 3}

Select a Lyapunov-Krasovskii function as

$$
\begin{aligned}
\Pi(x(k), k)= & 2 \sum_{i=1}^{k-2}\left[\|\lambda M x(i)\|^{2}-\|\lambda h(x(i))\|^{2}\right] \\
& +x^{\mathrm{T}}(k) \tilde{\Lambda} x(k)+x^{\mathrm{T}}(k-1) S x(k-1) \\
& +\|\lambda M x(k-1)\|^{2}-\|\lambda h(x(k-1))\|^{2} \\
\Delta \Pi(x(k), k)= & \Pi(x(k+1), k+1)-\Pi(x(k), k) \\
= & x^{\mathrm{T}}(k+1) \tilde{\Lambda} x(k+1)-x^{\mathrm{T}}(k) \tilde{\Lambda} x(k) \\
& +2 \sum_{i=1}^{2}\left[\|\lambda M x(i)\|^{2}-\|\lambda h(x(i))\|^{2}\right] \\
& +x^{\mathrm{T}}(k) S x(k)-x^{\mathrm{T}}(k-1) S x(k-1) \\
= & \xi^{\mathrm{T}}(k) \Psi_{1} \xi(k)+\mu_{1}^{2}\|w(k)\|^{2}
\end{aligned}
$$


where

$$
\xi(k)=\left[x^{\mathrm{T}}(k), x^{\mathrm{T}}(k-1), h^{\mathrm{T}}(x(k)), h^{\mathrm{T}}(x(k-1)), w^{\mathrm{T}}(k)\right]^{\mathrm{T}},
$$

743 and

$$
\Psi_{1}=\left[\begin{array}{ccccc}
Q_{5} & A_{1}^{\mathrm{T}} \tilde{\Lambda} A_{2} & A_{1}^{\mathrm{T}} \tilde{\Lambda} B_{1} & A_{1}^{\mathrm{T}} \tilde{\Lambda} B_{2} & A_{1}^{\mathrm{T}} \tilde{\Lambda} E \\
* & Q_{6} & A_{2}^{\mathrm{T}} \tilde{\Lambda} B_{1} & A_{2}^{\mathrm{T}} \tilde{\Lambda} B_{2} & A_{2}^{\mathrm{T}} \tilde{\Lambda} E \\
* & * & Q_{7} & B_{1}^{\mathrm{T}} \tilde{\Lambda} B_{2} & B_{1}^{\mathrm{T}} \tilde{\Lambda} E \\
* & * & * & Q_{8} & B_{2}^{\mathrm{T}} \tilde{\Lambda} B_{2} \\
* & * & * & * & Q_{9}
\end{array}\right]
$$

in which $Q_{5}=A_{1}^{\mathrm{T}} \tilde{\Lambda} A_{1}-\tilde{\Lambda}+S+\lambda^{2} M^{\mathrm{T}} M, Q_{6}=A_{2}^{\mathrm{T}} \tilde{\Lambda} A_{2}-$ $S+\lambda^{2} M^{\mathrm{T}} M, Q_{7}=B_{1}^{\mathrm{T}} \tilde{\Lambda} B_{1}-\lambda^{2} I, Q_{8}=B_{2}^{\mathrm{T}} \tilde{\Lambda} B_{2}-$ $\lambda^{2} I, Q_{9}=E^{\mathrm{T}} \tilde{\Lambda} E-\mu_{1}^{2} I$. Using the Schur complement, we have $\Psi_{1}<\operatorname{diag}\left[-\mu_{2}^{2} T, 0,0,0,0\right] \Leftrightarrow \Psi<0$. With the formulation in (48), there is

$$
\Delta \Pi(x(k), k) \leq-\mu_{2}^{2} e^{\mathrm{T}}(k) \Phi e(k)+\mu_{1}^{2}\|w(k)\|^{2} .
$$

Thus, $\Delta \Pi(x(k), k)<0$, if $e^{\mathrm{T}}(k) \Phi e(k)>\mu_{2}^{-2} \mu_{1}^{2}\|w(k)\|^{2}$ holds. Therefore for any $e(k)$, it can be verified that the PDF tracking error is bounded, i.e.

$$
e^{\mathrm{T}}(k) \Phi e(k) \leq \max \left\{e^{\mathrm{T}}(0) \Phi e(0), \mu_{2}^{-2} \mu_{1}^{2}\|w(k)\|^{2}\right\}
$$

which also implies that the controlled system is stable.

\section{REFERENCES}

[1] K. J. Astrom, Introduction to Stochastic Control Theory. New York: New York Academic, 1970.

[2] B. S. Chen and W. H. Zhang, "Stochastic control with state-dependent noise," IEEE Trans. on Automatic Control, vol. 49, pp. 45-57, 2004.

[3] M. Zyskowski, M. Sain, and R. Diersing, "Weighted least-squares, cost density-shaping, stochastic optimal control: A step towards total probabilistic control design," IEEE Conf. on Decision and Control, 2010.

[4] E. B. L. Andrew and X. Y. Zhou, "Stochastic optimal lqr control with integral quadratic constraints and indefinite control weights," IEEE Trans. on Automatic Control, vol. 44, pp. 1359-1369, 1999.

[5] L. Odhner and H. H. Asada, "Kalman filter for inhomogeneous population Markov chains with application to stochastic recruitment control of muscle actuators," 2010 American Control Conference Marriott Waterfront Baltimore, MD, USA, 2010.

[6] H. B. Ji and H. S. Xi, "Adaptive output-feedback policy for nonlinear stochastic systems," IEEE Trans. on Automatic Control, vol. 51, pp. 355-360, 2006.

[7] D. W. Stroock, An Introduction to Markov Processes. World Publishing Company, 2009.

[8] H. N. Wu and K. Y. Cai, "Model-independent robust stabilization for uncertain Markovian jump nonlinear systems via fuzzy control," IEEE Trans Systems, Man, and Cybernetics, Part B: Cybernetics, vol. 36, pp. 509-519, 2005.

[9] H. Y. Wu, L. Cao, and J. Wang, "Gray-box modelling and control of polymer molecular weight distribution using orthogonal polynomial neural networks,' Journal of Process Control, vol. 22, pp. 1624-1636, 2012.

[10] H. Yue, H. Wang, and J. Zhang, "Shaping of molecular weight distribution by iterative learning probability density function control strategies," Proc. IMechE Pt I: J. Syst. Contr. Eng., vol. 222, pp. 639-653, 2008.

[11] H. Wang, Bounded Dynamic Stochastic Systems: Modeling and Control. London: Springer-Verlag, 2000.

[12] H. Gommeren, D. Heitzmann, J. Moolenaar, and B. Scarlett, "Modelling and control of a jet mill plant," Powder Tech., vol. 108, pp. 147-154, 2000 .

[13] Z. K. Nagy and R. D. Braatz, "Advances and new directions in crystallization control," Annu. Rev. Chem. Biomol. Eng., vol. 3, pp. 55$75,2012$.

[14] T. Li, G. Li, and Q. Zhao, "Adaptive fault-tolerant stochastic shape control with application to particle distribution control," IEEE Trans. Systems, Man, and Cybernetics: Systems, vol. 45, no. 12, pp. 1592 1604, 2015.
[15] X. Sun, H. Yue, and H. Wang, "Modelling and control of the flame temperature distribution using probability density function shaping,' Trans. Inst. Measurement and Control, vol. 28, pp. 401-428, 2006.

[16] J. Zhou, H. Yue, J. Zhang, and H. Wang, "Iterative learning double closed-loop structure for modeling and controller design of output stochastic distribution control systems," IEEE Trans. Control System Technology, vol. 22, pp. 2261-2276, 2014.

[17] A. E. Abharian and A. H. Fadaei, "Power probability density function control and performance assessment of a nuclear research reactor," Annals of Nuclear Energy, vol. 64, pp. 11-20, 2014.

[18] M. G. Forbes and J. F. Forbes, "Control design for first-order processes: Shaping the probability density of the process state,' Journal of Process Control, vol. 14, pp. 399-410, 2004.

[19] M. Karny and T. Kroupa, "Axiomatisation of fully probabilistic design," Information Sciences, vol. 186, pp. 105-113, 2012.

[20] J. Zhou and H. Wang, "Optimal tracking control of output PDF: mean square root b-spline model," Control Theory and Application (in Chinese), vol. 22, pp. 369-376, 2005.

[21] H. Yue, J. Zhou, and H. Wang, "Minimum entropy control of b-spline PDF systems with mean constraint," Automatica, vol. 42, pp. 989-994, 2006.

[22] H. Y. Chen and H. Wang, "The system with parameter random variation PDF control based on LMI," Acta Automatica Sinica, vol. 33, pp. 12161220, 2007.

[23] H. Wang and P. Afshar, "Ilc-based fixed-structure controller design for output PDF shaping in stochasitic systems using LMI techniques," IEEE Trans. on Automatic Control, vol. 54, pp. 760-773, 2009.

[24] J. F. Zhang, H. Yue, and J. L. Zhou, "Predictive PDF control in shaping of molecular weight distribution based-on a new modelling algorithm," Journal of Process Control, vol. 30, pp. 80-89, 2015.

[25] A. Ferramosca, D. Limon, A. Gonzlez, D. Odloak, and E. Camacho, "MPC for tracking zone regions," Journal of Process Control, vol. 20, pp. $506-516,2010$.

[26] F. V. Lima and C. Georgakis, "Design of output constraints for modelbased non-square controllers using interval operability," Journal of Process Control, vol. 18, pp. 610 - 620, 2008.

[27] A. H. González and D. Odloak, "A stable MPC with zone control," Journal of Process Control, vol. 19, pp. 110 - 122, 2009.

[28] Z. Xu, J. Zhao, and J. Qian, "Zone model predictive control algorithm using soft constraint method," Machine Tool and Hydraulics, vol. 12, pp. 106-108, 2004.

[29] S. Ding, Model-based Fault Diagnosis Techniques. Berlin: Springer, 2008.

[30] P. M. Frank, S. X. Ding, and T. Marcu, "Model-based fault diagnosis in technical processes," Transactions of the Institute of Measurement and Control, vol. 22, pp. 57-101, 2000.

[31] R. Isermann, Fault-Diagnosis Systems: An Introduction from Fault Detection to Fault Tolerance. Berlin: Springer, 2006.

[32] R. Patton, P. M. Frank, and R. Clark, Issues of Fault Diagnosis for Dynamic Systems. Berlin: Springer, 2000.

[33] V. Venkatasubramanian, R. Rengaswamy, K. Yin, and S. N. Kavuri, "A review of process fault detection and diagnosis. Part I: Quantitative model-based methods," Computers and Chemical Engineering, vol. 27, pp. 293-311, 2003.

[34] B. Jiang and F. N. Chowdhury, "Fault estimation and accommodation for linear MIMO discrete time systems," IEEE Trans. on Control System Technology, vol. 13, pp. 493-499, 2005.

[35] J. Lunze and T. Steffen, "Control reconfiguration after actuator failures using disturbance decoupling methods," IEEE Trans. on Automatic Control, vol. 51, pp. 1590-1601, 2006.

[36] J. Richter, Reconfigurable Control of Nonlinear Dynamical Systems: A Fault-hiding Approach. Springer, 2011.

[37] M. M. Seron, J. A. D. Donha, and J. Richter, "Fault tolerant control using virtual actuators and set-separation detection principles," International Journal of Robust and Nonlinear Control, vol. 22, pp. 709-742, 2012.

[38] T. Steffen, Control Reconfiguration of Dynamical Systems. Springer, 2005.

[39] Y. Wang, D. Zhao, Y. Li, and S. X. Ding, "Unbiased minimum variance fault and state estimation for linear discrete time-varying twodimensional systems," IEEE Trans. Automatic Control, 2017.

[40] D. Zhao, Y. Wang, Y. Li, and S. X. Ding, "H fault estimation for 2 d linear discrete time-varying systems based on krein space method," IEEE Trans. Systems, Man, and Cybernetics: Systems, 2017.

[41] Z. Gao, H. Wang, and T. Chai, "A robust fault detection filtering for stochastic distribution systems via descriptor estimator and parametric gain design," IET Control Theory Appllication, vol. 1, pp. 1286-1293, 2007.
796 
[42] H. J. Ma and G. H. Yang, "Observer-based fault diagnosis for a class of nonlinear multiple input multiple output uncertain stochastic systems using B-spline expansions," IET Control Theory Appllication, vol. 5, pp. 173-187, 2011.

[43] H. Wang and W. Lin, "Applying observer based FDI techniques to detect faults in dynamic and bounded stochastic distributions," International Journal of Control, vol. 73, pp. 1424-1436, 2000.

[44] L. N. Yao, J. F. Qin, H. Wang, and B. Jiang, "Design of new fault diagnosis and fault tolerant control scheme for non-Gaussian singular stochastic distribution systems," Automatica, vol. 48, pp. 2305-2313, 2012.

[45] L. Yao and L. Feng, "Fault diagnosis and fault tolerant tracking control for the non-Gaussian singular time-delayed stochastic distribution system with PDF approximation error," Neurocomputing, vol. 175, pp. 538-543, 2016.

[46] Y. Tang, J. L. Zhou, and J. Wang, "Tolerant tracking controller design with guaranteed success rate of output sdc systems," The 27th Chinese Control and Decision Conference (CCDC), pp. 2724-2729, 2015.

[47] L. Guo and H. Wang, Stochastic Distribution Control Systems Design: A Convex Optimization Approach. Springer-Verlag Ltd., 2010.

[48] L. N. Yao, A. P. Wang, and H. Wang, "Fault detection, diagnosis and tolerant control for non-Gaussian stochastic distribution systems using a rational square-root approximation model," International Journal of Modeling, Identification and Control, vol. 3, pp. 162-172, 2008.

[49] S. X. Ding, "Integrated design of feedback controllers and fault detectors," Annual Reviews in Control, vol. 33, pp. 124-135, 2009.

[50] J. Lan and R. J. Patton, "A new strategy for integration of fault estimation within fault-tolerant control," Automatica, vol. 69, pp. 48-59, 2016.

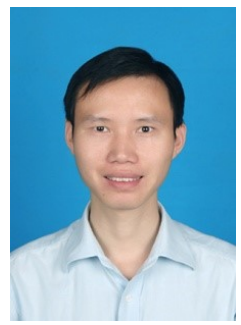

Jinglin Zhou received the BEng, MSc and Ph.D. degrees from Daqing Petroleum Institute, Hunan University, and the Institute of Automation, Chinese Academy of Sciences, in 1999, 2002 and 2005, respectively. He was an Academic Visitor at Department of Automatic Control and Systems Engineering, the University of Sheffield. Dr. Zhou is currently a professor in the College of Information Science and Technology, Beijing University of Chemical Technology. His research interests include stochastic distribution control, fault detection and diagnosis, variable structure control and their applications.

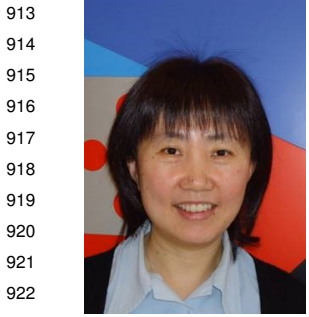

Hong Yue (M99, S04) received the B.Eng. and the M.Sc. degrees in process control engineering from Beijing University of Chemical Technology, Beijing, China in 1990 and 1993, respectively, and the Ph.D. degree in control theory and applications from East China University of Science and Technology, Shanghai, China in 1996. She is a senior lecturer at the Department of Electronic and Electrical Engineering, University of Strathclyde. Her research interests are in systems and control with a focus on modelling, control and optimisation of complex systems. 